Pacific

Journal of

Mathematics

THE GENERALIZED BEREZIN TRANSFORM AND COMMUTATOR IDEALS

Kenneth R. DAVIDSON And Ronald G. Douglas

Volume 222 No. 1

November 2005 


\title{
THE GENERALIZED BEREZIN TRANSFORM AND COMMUTATOR IDEALS
}

\author{
Kenneth R. Davidson And Ronald G. Douglas
}

\begin{abstract}
For a quasi-free module over a function algebra $A(\Omega)$, we define an analogue of the Berezin transform and relate this to the quotient of the $C^{*}$ algebra it generates modulo the commutator ideal.
\end{abstract}

Certainly the best understood commutative Banach algebras are those consisting of all continuous complex-valued functions on a compact Hausdorff space. Indeed, most self-adjoint phenomena involving them have been thoroughly investigated. In particular, the study of their representation theory as operators on a Hilbert space, which is essentially the spectral theory for normal operators, shows that such representations are defined by multiplication on $L^{2}$-spaces. Over the past few decades, other classes of operators have been introduced that are defined by functions, but which involve more complicated methods; for example, Toeplitz operators and pseudo-differential operators. In both cases, one shows that the operators so defined behave like the functions used to define them, up to operators of lower order.

To be more precise, let $H^{2}(\mathbb{D})$ be the Hardy space of functions in $L^{2}(\mathbb{T})$ consisting of the functions with zero negative Fourier coefficients, and let $P$ be the projection of $L^{2}(\mathbb{T})$ onto $H^{2}(\mathbb{D})$. The Toeplitz operator $T_{\varphi}$ for the function $\varphi$ in $L^{\infty}(\mathbb{T})$ is defined, on $H^{2}(\mathbb{D})$, to be pointwise multiplication by $\varphi$ followed by $P$. If $\mathfrak{T}$ denotes the $\mathrm{C}^{*}$-algebra generated by the Toeplitz operators $T_{\varphi}$, for $\varphi$ a continuous function on $\mathbb{T}$, then $\mathfrak{T}$ contains the algebra $\mathfrak{K}$ of compact operators, and the quotient algebra $\mathfrak{T} / \mathfrak{K}$ is isometrically isomorphic to $C(\mathbb{T})$. If, instead of $H^{2}(\mathbb{D})$, we take the Bergman space $B^{2}(\mathbb{D})$ consisting of the functions in $L^{2}(\mathbb{D})$ which are almost everywhere equal to a holomorphic function on $\mathbb{D}$, then we can define "Toeplitz operators" analogously, and the $\mathrm{C}^{*}$-algebra $\mathfrak{T}^{\prime}$ generated by the operators defined by continuous functions on the closed unit disk, contains $\mathfrak{K}$ and the quotient algebra $\mathfrak{T}^{\prime} / \mathfrak{K}$ is again isometrically isomorphic to $C(\mathbb{T})$.

The description of these two examples can be carried over to the case of several variables in more than one way. First, consider the boundary $\partial \mathbb{B}^{n}$ of the unit ball

MSC2000: 46L06, 47L05, 47L15, 47L20, 47L80.

Keywords: Toeplitz operators, Toeplitz $\mathrm{C}^{*}$-algebras, Hilbert modules, Berezin transform, commutator ideals in $\mathrm{C}^{*}$-algebras. 
$\mathbb{B}^{n}$ in $\mathbb{C}^{n}$ and the Hardy space of functions in $L^{2}\left(\partial \mathbb{B}^{n}\right)$ that have holomorphic extensions to the ball. Again, we can define Toeplitz operators using functions defined on $\partial \mathbb{B}^{n}$ and the $\mathrm{C}^{*}$-algebra generated by the ones defined by continuous functions again contains the compact operators and the quotient algebra is isometrically isomorphic to $C\left(\partial \mathbb{B}^{n}\right)$. Second, for the corresponding Bergman space we can define Toeplitz operators for functions on the closed ball and the $\mathrm{C}^{*}$-algebra generated by the continuous functions contains the compact operators and again, the quotient algebra is isometrically isomorphic to $C\left(\partial \mathbb{B}^{n}\right)$. Thirdly, if we consider the polydisk $\mathbb{D}^{n}$ and the Hardy subspace of $L^{2}\left(\mathbb{T}^{n}\right)$, then although the $C^{*}$-algebra generated by the Toeplitz operators defined by continuous functions on $\mathbb{T}^{n}$ contains the ideal of compact operators $\mathfrak{K}$, in this case $\mathfrak{K}$ is not the commutator ideal $\mathfrak{C}$ in $\mathfrak{T}$. But $\mathfrak{C}$ is proper and the quotient algebra is isometrically isomorphic to $C\left(\mathbb{T}^{n}\right)$.

The phenomenon we consider in this note concerns an algebra of operators defined by functions, the $\mathrm{C}^{*}$-algebra that the algebra generates and the quotient algebra defined modulo the commutator ideal. The results we obtain will contain all the examples above and show that, in a certain sense, it is the domain that is important, rather than the particular Hilbert space on which the operators are defined. We will accomplish this by generalizing the notion of a transform introduced by Berezin [1972] in connection with quantization. In our approach, the Hilbert space is closely related to the algebra of holomorphic functions on the domain and involves a notion of kernel Hilbert space that is a module over the algebra.

We build on results of several authors who have studied the Berezin transform. In particular, there is the result of McDonald and Sundberg [1979] concerning the nature of the transform for the $\mathrm{C}^{*}$-algebra of Toeplitz operators defined by bounded holomorphic multipliers on the Bergman space. Some of our proofs are closely related to arguments in [McDonald et al. 1979]. Further, some issues we study overlap questions raised by Arveson [1969].

We also relate a further study of Sundberg [1987] on the $C^{*}$-algebras generated by Toeplitz-like operators to our results including a relation between the property of a module multiplier having closed range to the behavior of its Gelfand transform.

Finally, we study the relationship between the kernel ideal of the generalized Berezin transform and the commutator ideal.

\section{Definition and basic properties}

Regardless of the original motivation of Berezin [1972] for introducing it, the Berezin transform essentially provides a kind of "symbol" for certain natural operators on Hilbert spaces of holomorphic functions. For our generalized transform, we will use kernel Hilbert spaces over bounded domains in $\mathbb{C}^{n}$, which are also contractive Hilbert modules for natural function algebras over the domain. More 
precisely, we use the concept introduced in [Douglas and Misra 2003] for the study of module resolutions.

For $\Omega$ a bounded domain in $\mathbb{C}^{n}$, let $A(\Omega)$ be the function algebra obtained as the completion of the set of functions that are holomorphic in some neighborhood of the closure of $\Omega$. For $\Omega$ the unit ball $\mathbb{B}^{n}$ or the polydisk $\mathbb{D}^{n}$ in $\mathbb{C}^{n}$, we obtain the familiar ball and polydisk algebras $A\left(\mathbb{B}^{n}\right)$ and $A\left(\mathbb{D}^{n}\right)$, respectively. The Hilbert space $M$ is said to be a contractive Hilbert module over $A(\Omega)$ if $M$ is a unital module over $A(\Omega)$ with module map $A(\Omega) \times M \rightarrow M$ such that

$$
\|\varphi f\|_{\mathcal{M}} \leq\|\varphi\|_{A(\Omega)}\|f\|_{\mathcal{M}} \quad \text { for } \quad \varphi \in A(\Omega) \quad \text { and } \quad f \in \mathcal{M} .
$$

The space $\mathscr{R}$ is said to be a quasi-free Hilbert module of rank $m$ over $A(\Omega)$, where $1 \leq m \leq \infty$, if it is obtained as the completion of an inner product on the algebraic tensor product $A(\Omega) \otimes \ell_{m}^{2}$ such that

(1) eval $_{z}: A(\Omega) \otimes \ell_{m}^{2} \rightarrow \ell_{m}^{2}$, the canonical evaluation map at the point $z$, is bounded for $z$ in $\Omega$ and locally uniformly bounded on $\Omega$;

(2) $\left\|\Sigma \varphi \theta_{i} \otimes x_{i}\right\|_{\mathscr{R}} \leq\|\varphi\|_{A(\Omega)}\left\|\Sigma \theta_{i} \otimes x_{i}\right\|_{\mathscr{R}}$ for $\varphi,\left\{\theta_{i}\right\}$ in $A(\Omega)$ and $\left\{x_{i}\right\}$ in $\ell_{m}^{2}$; and

(3) for $\left\{F_{i}\right\}$ a sequence in $A(\Omega) \otimes \ell_{m}^{2}$ Cauchy in the $\mathscr{R}$-norm, it follows that $\operatorname{eval}_{z}\left(F_{i}\right) \rightarrow 0$ for all $z$ in $\Omega$ if and only if $\left\|F_{i}\right\|_{\mathscr{R}} \rightarrow 0$.

Here, $\ell_{m}^{2}$ is the $m$-dimensional Hilbert space.

In [Douglas and Misra 2003], another characterization and other properties of quasi-free Hilbert modules are given. This concept is closely related to the notions of sharp and generalized Bergman kernels studied in [Curto and Salinas 1984; Agrawal and Salinas 1988; Salinas 1988].

Although much of what follows is valid for the case of $m=\infty$, we confine our attention here to the finite-rank case. Hence the evaluation function eval ${ }_{z}: \mathscr{R} \rightarrow \ell_{m}^{2}$, which is defined to be the extension of evaluation on $A(\Omega) \otimes \ell_{m}^{2}$, is onto and an $\mathscr{R}$-valued holomorphic function in $z$. If eval* $: \ell_{m}^{2} \rightarrow \mathscr{R}$ is the operator adjoint, then it is bounded below and is anti-holomorphic in $z$. Moreover, the lower bound is continuous in $z$ since $\operatorname{eval}_{z}$ eval $_{z}^{*}$ is a real-analytic function in $\boldsymbol{z}$.

The following lemma provides some well known connections between eval $z$, module multiplication, and the kernel function. Let $M_{\varphi}$ denote the operator on $\mathscr{R}$ defined by module multiplication.

Lemma 1.1. For $z$ in $\Omega$ and $\varphi$ in $A(\Omega)$, one has

$$
M_{\varphi}^{*} \operatorname{eval}_{z}^{*}=\bar{\varphi}(\boldsymbol{z}) \operatorname{eval}_{z}^{*} \quad \text { and } \quad \operatorname{eval}_{z} M_{\varphi}=\varphi(z) \operatorname{eval}_{z}
$$

Moreover,

$$
K(\boldsymbol{w}, \boldsymbol{z})=\operatorname{eval}_{\boldsymbol{w}} \mathrm{eval}_{z}^{*}: \Omega \times \Omega \rightarrow \mathfrak{L}\left(\ell_{m}^{2}\right)
$$

is the kernel function for $\mathscr{R}$. 
Proof. The two identities in the first sentence follow from the fact that

$$
\left(\operatorname{eval}_{z} M_{\varphi}\right) f=\operatorname{eval}_{\boldsymbol{z}}(\varphi f)=\varphi(\boldsymbol{z}) f(\boldsymbol{z})=\varphi(\boldsymbol{z}) \operatorname{eval}_{\boldsymbol{z}} f
$$

for $\varphi \in A(\Omega), f \in \mathscr{R}$ and $z \in \Omega$. For the rest, take $\boldsymbol{w}$ and $z$ in $\Omega$ and $x$ in $\ell_{m}^{2}$. The function $f(\boldsymbol{w})=K(\boldsymbol{w}, \boldsymbol{z}) x$ is in $\mathscr{R}$ and

$$
\langle g, f\rangle_{\mathscr{R}}=\langle g, K(\cdot, \boldsymbol{z}) x\rangle_{\Re}=\langle g(\boldsymbol{z}), x\rangle_{\ell_{m}^{2}} \quad \text { for } \quad g \in \mathscr{R}
$$

by the definition of the kernel function $K$. Since $g(\boldsymbol{z})=\operatorname{eval}_{z} g$, we have

$$
\langle g(z), x\rangle=\left\langle\operatorname{eval}_{z} g, x\right\rangle=\left\langle g, \operatorname{eval}_{z}^{*} x\right\rangle_{\Re}
$$

and therefore, $K(\cdot, \boldsymbol{z}) x=\operatorname{eval}_{z}^{*} x$. Applying $\operatorname{eval}_{\boldsymbol{w}}$ to both sides we get, as desired,

$$
K(\boldsymbol{w}, \boldsymbol{z}) x=\operatorname{eval}_{\boldsymbol{w}} \operatorname{eval}_{z}^{*} x \quad \text { for } x \in \ell_{m}^{2} .
$$

To define the transform we need the polar form of evaluation on $\mathscr{R}$. Thus, set eval $_{z}^{*}=V_{z} Q_{z}$, where

(1) $V_{z}$ is an isometry from $\ell_{m}^{2}$ into $\mathscr{R}$ which is real-analytic in $z$, and

(2) $Q_{z}$ is a positive, invertible operator on $\ell_{m}^{2}$, which is also real-analytic in $z$.

The properties of eval $z$ in Lemma 1.1 carry over to $V_{z}$ to yield the following results:

Lemma 1.2. For $\varphi$ in $A(\Omega)$ and $\boldsymbol{z}$ in $\Omega$, it follows that

$$
M_{\varphi}^{*} V_{z}=\overline{\varphi(z)} V_{z} \quad \text { and } \quad V_{z}^{*} M_{\varphi}=\varphi(z) V_{z}^{*} .
$$

We can now define the Generalized Berezin Transform (GBT) on $\mathscr{R}$.

Definition 1.3. If $\mathscr{R}$ is a quasi-free Hilbert module of finite rank over $A(\Omega)$, then the Generalized Berezin Transform $\Gamma$ maps $\mathfrak{L}(\mathscr{R})$ to $\mathrm{C}_{b}\left(\Omega, \mathfrak{L}\left(\ell_{m}^{2}\right)\right)$, the space of bounded continuous $m \times m$ matrix-valued functions on $\Omega$, by $(\Gamma X)(z)=V_{z}^{*} X V_{z}$ for $X$ in $\mathfrak{L}(\mathscr{R})$ and $z$ in $\Omega$.

Lemma 1.4. The GBT has the following elementary properties:

(1) $\Gamma$ is contractive: $\|(\Gamma X)(z)\|_{\mathfrak{L}\left(\ell_{m}^{2}\right)} \leq\|X\|_{\mathfrak{L}(\mathscr{R})}$ for $X \in \mathfrak{L}(\mathscr{R})$ and $\boldsymbol{z} \in \Omega$.

(2) $\Gamma$ is linear: $\Gamma\left(a_{1} X_{1}+a_{2} X_{2}\right)=a_{1} \Gamma\left(X_{1}\right)+a_{2} \Gamma\left(X_{2}\right)$ for $a_{1}, a_{2} \in \mathbb{C}$ and $X_{1}, X_{2} \in$ $\mathfrak{L}(\mathscr{R})$.

(3) $\Gamma$ is self-adjoint: $(\Gamma X)(\boldsymbol{z})^{*}=\Gamma\left(X^{*}\right)(\boldsymbol{z})$ for $X \in \mathfrak{L}(\mathscr{R})$ and $\boldsymbol{z} \in \Omega$.

(4) $\Gamma(X)(z)$ is continuous (indeed real analytic) on $\Omega$.

If $V_{z}$ is any isometry-valued function from $\Omega$ to $\mathfrak{L}\left(\ell_{m}^{2}, \mathscr{R}\right)$, one could define a GBT having the properties in this lemma but little more. The interest in our setting comes about because of the relationship between the GBT and the operators defined by the algebra $A(\Omega)$. In general, one is interested in the behavior of the GBT on the $\mathrm{C}^{*}$-algebra generated by the operators defined by module multiplication or the 
corresponding algebra obtained when $A(\Omega)$ is replaced by a larger, natural function algebra of bounded holomorphic functions (see Section 5).

The following proposition is easily deduced from Lemma 1.2.

Proposition 1.5. For $\varphi, \psi$ in $A(\Omega)$ and $X$ in $\mathfrak{L}(\mathscr{R})$ it follows that:

(1) $\Gamma\left(M_{\varphi} X\right)(\boldsymbol{z})=\varphi(\boldsymbol{z})(\Gamma X)(\boldsymbol{z})$ and $\Gamma\left(X M_{\varphi}^{*}\right)(\boldsymbol{z})=\bar{\varphi}(\boldsymbol{z})(\Gamma X)(\boldsymbol{z})$ for $\boldsymbol{z}$ in $\Omega$,

(2) $\Gamma\left(M_{\varphi}\right)=\varphi$ and $\Gamma\left(M_{\varphi}^{*}\right)=\bar{\varphi}$, and

(3) $\Gamma\left(M_{\varphi} M_{\psi}^{*}\right)=\varphi \bar{\psi}$.

These results show that the "symbol" defined by the GBT agrees with the multiplier for operators defined by module multiplication for functions in $A(\Omega)$ or their complex conjugates. Also, it is multiplicative on products of the form $\varphi \bar{\psi}$ for $\varphi$ and $\psi$ in $A(\Omega)$; but it is easy to check that $\Gamma\left(M_{\varphi} M_{\psi}^{*}\right) \neq \Gamma\left(M_{\psi}^{*} M_{\varphi}\right)$, in general. This comes down to evaluating $\Gamma$ on commutators of the form $\left[M_{\varphi}, M_{\psi}^{*}\right]$ for $\varphi, \psi$ in $A(\Omega)$.

If one considers the classical examples where $\mathscr{R}$ is either the Hardy or Bergman modules on the unit disk $\mathbb{D}$, one sees that the Berezin transform is nicely-behaved for the $\mathrm{C}^{*}$-algebra generated by module multipliers only on the boundary of $\mathbb{D}$ and vanishes there on the commutator ideal. More interesting is the behavior of the Berezin Transform on the corresponding algebra defined by module multiplication by the algebra $H^{\infty}(\mathbb{D})$ of bounded holomorphic functions on $\mathbb{D}$ which we'll consider in Section 5.

To get started, we consider the basic relationship between the operators defined by module multiplication and the quotient algebra defined by the commutator ideal. Understanding this quotient algebra is equivalent to characterizing the multiplicative linear functionals on the algebra.

Let $\mathfrak{T}(\mathscr{R})$ denote the $\mathrm{C}^{*}$-algebra generated by $\left\{M_{\varphi}: \varphi \in A(\Omega)\right\}$ acting on $\mathscr{R}$ and $\mathfrak{C}(\mathscr{R})$ be the closed two-sided ideal in $\mathfrak{T}(\mathscr{R})$ generated by the commutators in $\mathfrak{T}(\mathscr{R})$. Then $\mathfrak{T}(\mathscr{R}) / \mathfrak{C}(\mathscr{R})$ is a commutative $\mathrm{C}^{*}$-algebra, possibly $(0)$, and hence is isometrically isomorphic to $C\left(X_{\mathscr{R}}\right)$ for some compact Hausdorff space $X_{\mathscr{R}}$. Thus the points of $X_{\mathscr{R}}$ are precisely the characters on $\mathfrak{T}(\mathscr{R})$. So we call $X_{\mathscr{R}}$ the character space of $\mathscr{R}$. Our first result relates the character space to the maximal ideal space $M_{A}$ of $A(\Omega)$.

Theorem 1.6. For $\mathscr{R}$ a quasi-free Hilbert module of finite rank, the map taking $\varphi$ to $M_{\varphi}+\mathfrak{C}(\mathscr{R})$ extends to a surjective $*$-homomorphism $\tau: C\left(M_{A}\right) \rightarrow \mathfrak{T}(\mathscr{R}) / \mathfrak{C}(\mathscr{R})=$ $C\left(X_{\mathscr{R}}\right)$. Consequently one can identify $X_{\mathscr{R}}$ with a closed subset of $M_{A}$.

Proof. If $\mathfrak{C}(\mathscr{R})=\mathfrak{T}(\mathscr{R})$ whence $X_{\mathscr{R}}$ is empty, then $\tau$ is the zero map and the result is vacuous; hence we assume that $X_{\mathscr{R}}$ is nonempty. Also, we abbreviate $A=A(\Omega)$ in the following proof. 
Consider the diagram

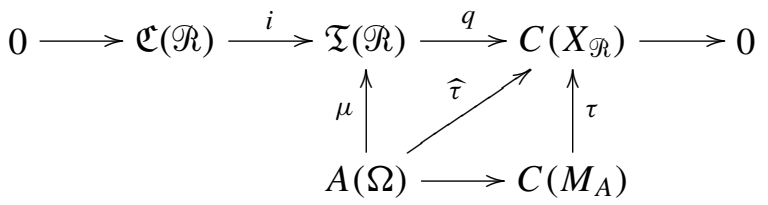

where the map $\mu$ from $A(\Omega)$ to $\mathfrak{T}(A)$ sends $\varphi$ to $M_{\varphi}$, the map from $A(\Omega)$ to $C\left(M_{A}\right)$ is the Gelfand map, and $\widehat{\tau}=q \mu$ is defined by composition. Then $\tau$ exists by the universal properties of the Gelfand map. Moreover, since the image of $A(\Omega)$ in $\mathfrak{T}(A)$ generates it, the range of $\widehat{\tau}$ generates $C\left(X_{\mathscr{R}}\right)$. Therefore, the range of $\tau$ generates $C\left(X_{\mathscr{R}}\right)$ and hence separates the points of $X_{\mathscr{R}}$, which implies that we can identify $X_{\mathscr{R}}$ as a closed subset of $M_{A}$.

Actually, this holds for an arbitrary Hilbert module $\mathscr{R}$ over $A(\Omega)$. Although the map $\mu$ need not be contractive, the map $\widehat{\tau}$ will be.

As we mentioned above, for the Hardy and Bergman modules, the quotient algebras $\mathfrak{T}\left(H^{2}(\mathbb{D})\right) / \mathfrak{C}\left(H^{2}(\mathbb{D})\right)$ and $\mathfrak{T}\left(B^{2}(\mathbb{D})\right) / \mathfrak{C}\left(B^{2}(\mathbb{D})\right)$ are both equal to $C(\partial \mathbb{D})$. Hence, for both $\mathscr{R}=H^{2}(\mathbb{D})$ and $B^{2}(\mathbb{D})$, the character space is equal to the Šilov boundary. We will see in Example 4.5 that this is not always the case. An even more dramatic example will be given in Example 4.6 in which we show for a module which is similar to the Hardy module (but not contractive) that the character space can be empty. This is in striking contrast to the fact that for contractive modules over nice domains including the unit disk, the character space must contain the whole Šilov boundary. See Corollary 3.6.

\section{Pointed function algebras}

We introduce a property for points in the maximal ideal space of a function algebra which is related to the well known notions of peak point and p-point. We shall give a few cases where this property can be explicitly exhibited. When this work was close to completion, we asked John Wermer if he could establish this property in certain examples. The result is a complete answer [Izzo 2005] that reduces our notion to the classical ones.

Definition 2.1. The function algebra $A$ is said to be pointed at $\alpha$ in $M_{A}$ if $A$ is the closed linear span of the set $\left\{\varphi \in A:|\varphi(\alpha)|=\|\varphi\|_{A}\right\}$. A function algebra $A$ is said to be pointed if it is pointed on a dense subset of the Šilov boundary $\partial A$ of $A$. We will say that a domain $\Omega$ is pointed if $A(\Omega)$ is pointed.

Since the set of functions achieving their maxima at a point $\alpha$ is closed under multiplication, the closed linear span is automatically a closed subalgebra of $A$. 
Recall that a point $\alpha$ in $M_{A}$ is a p-point if for every neighbourhood $U$ of $\alpha$, there is a function $\varphi$ in $A$ which attains its maximum modulus at $\alpha$ and nowhere outside of $U$. It is a peak point if there is a function $\varphi$ which attains its maximum precisely at $\alpha$. In the metric case, it is well known that p-points are peak points.

The following easy lemma shows that being pointed implies being a p-point.

Lemma 2.2. If function algebra $A$ is pointed at $\alpha$, then $\alpha$ is a p-point. In the metrizable case, it is a peak point.

Proof. If $U$ is a neighborhood of $\alpha$, for each point $\beta$ in $M_{A} \backslash U$, choose a function $f$ in $A$ such that $\|f\|=f(\alpha)=1$ and $f(\beta) \neq 1$. It is easy to modify $f$ so that its range meets the unit circle only at 1 . A finite cover yields a finite family of such functions $f_{i}$, where $1 \leq i \leq k$, so that for each $\beta$ in $M_{A} \backslash U$, there is an $i$ with $\left|f_{i}(\beta)\right|<1=f_{i}(\alpha)=\left\|f_{i}\right\|$. The average of the $f_{i}$ peaks at $\alpha$ and has modulus less than 1 on $M_{A} \backslash U$. So $\alpha$ is a p-point.

The converse was obtained by Alex Izzo. It is a short but clever argument, and yields a much stronger result than we anticipated.

Theorem 2.3 [Izzo 2005]. If $\alpha$ is a p-point of a function algebra $A$, then $A$ is pointed at A. If $\alpha$ is a peak point, then every function in $A$ is the linear combination of two functions which peak precisely at $\alpha$.

In the metric case, the peak points form a dense $G_{\delta}$ of the Šilov boundary. In general, the p-points are dense (but perhaps not even Borel). See [Gamelin 1969]. Thus we obtain:

\section{Corollary 2.4. Every function algebra is pointed.}

Even though this general result eliminates the need to demonstrate that particular function algebras are pointed, it is of interest to see when this can be achieved in practice. So we provide a few examples.

Example 2.5. Consider finitely connected domains in $\mathbb{C}$ whose boundary consists of a finite union of Jordan curves. Let us begin with the case of the annulus

$$
\mathbb{A}=\{z \in \mathbb{C}: 0<r<|z|<R<\infty\} .
$$

Fix $\alpha$ on the outer circle. The functions $\left\{z^{n}\right\}, n \geq 0$, satisfy the requirement that $\left\|z^{n}\right\|=R^{n}=\left|\alpha^{n}\right|$. Moreover, the function $\varphi_{\lambda}(z)=z+\lambda z^{-n}$, for $n>0$ and $\lambda$ in $\mathbb{C}$, takes its maximum on either the inner or outer circles. For nonzero $\lambda$ of sufficiently small absolute value, the maximum is achieved on the outer circle. By choosing the phase of $\lambda$ correctly, call it $\lambda_{n}$, we can force the maximum absolute value of $\varphi_{\lambda_{n}}$ to occur at $\alpha$. Since the closed linear span of the set of functions $\left\{z^{n}, z+\lambda_{n} z^{-n}\right\}$ is $A(\mathbb{A})$, we see that $\mathbb{A}$ is pointed at each $\alpha$ on the outer circle. But being pointed is a conformal invariant in the sense that if $\Omega$ is pointed at $\alpha$ in $\partial \Omega$ 
and $\mu$ is a conformal self-map on $\Omega$ that extends to $\partial \Omega$, then $\Omega$ is also pointed at $\mu(\alpha)$. Therefore, $\mathbb{A}$ is a pointed domain as are all one-connected domains with Jordan curves as boundaries.

Now consider an arbitrary bounded finitely connected domain $\Omega$ in $\mathbb{C}$ with boundary consisting of Jordan curves. By making a conformal transformation on $\Omega$ which extends to $\partial \Omega$, we can assume the outer boundary is a circle. Let $\alpha$ be a point on the bounding outer circle of $\Omega$. If $z_{0}$ is a point inside this circle not in $\Omega$, we can repeat the above argument to exhibit a function $z+\lambda_{n}\left(z-z_{0}\right)^{-n}$ which peaks at $\alpha$. Doing this for a point in each bounded component of $\mathbb{C} \backslash \Omega$, we obtain a set of functions in $A(\Omega)$ each of which peaks at $\alpha$ and for which their closed linear span equals $A(\Omega)$. Therefore, $\Omega$ is a pointed domain.

Example 2.6. An easy argument shows that the product $\Omega_{1} \times \Omega_{2}$ in $\mathbb{C}^{m+n}$ of two pointed domains $\Omega_{1}$ in $\mathbb{C}^{m}$ and $\Omega_{2}$ in $\mathbb{C}^{n}$ is also pointed. The Šilov boundary of the product is the product of the Šilov boundaries. The key observations are (1) that the closed linear span of functions of the form $\varphi \psi$ for $\varphi$ in $A\left(\Omega_{1}\right)$ and $A\left(\Omega_{2}\right)$ generates $A\left(\Omega_{1} \times \Omega_{2}\right)$ and (2) that $\left|\varphi\left(\alpha_{1}\right) \psi\left(\alpha_{2}\right)\right|=\|\varphi \psi\|_{A\left(\Omega_{1} \times \Omega_{2}\right)}$ if $\left|\varphi\left(\alpha_{1}\right)\right|=\|\varphi\|_{A\left(\Omega_{1}\right)}$ and $\left|\psi\left(\alpha_{2}\right)\right|=\|\psi\|_{A\left(\Omega_{2}\right)}$. Therefore, the polydisk is pointed.

The following argument is not nearly as definitive as the Izzo result, but provides a rather different approach.

Proposition 2.7. The ball algebra $A\left(\mathbb{B}^{n}\right)$ is pointed.

Proof. Let $n=2$. Observe that the following functions peak at (1,0): $1, z_{1}, z_{1}^{2}+z_{2}^{2}$ and $z_{1}^{2}+z_{2}^{3}$. In addition, let $h \in A(\mathbb{D})$ with $\|h\|_{\infty} \leq 1$ and consider $f\left(z_{1}, z_{2}\right)=$ $\left(1+z_{1}\right) z_{1} / 2+\left(1-z_{1}\right) z_{2} h\left(z_{1}\right) / 2$. Then by Cauchy-Schwartz,

$$
\left|f\left(z_{1}, z_{2}\right)\right|^{2} \leq\left(\left|\frac{1+z_{1}}{2}\right|^{2}+\left|\frac{1-z_{1}}{2}\right|^{2}\right)\left(\left|z_{1}\right|^{2}+\left|z_{2}\right|^{2}\|h\|^{2}\right) \leq \frac{1}{2}\left(1+\left|z_{1}\right|^{2}\right) \leq 1 .
$$

So this function also peaks at $(1,0)$.

The span of all functions peaking at $(1,0)$ is an algebra. So this span includes all polynomials of the form $\sum_{i} h_{i}\left(z_{1}\right) z_{2}^{i}$ provided that $h_{1}(1)=0$. But the functional taking a polynomial to $h_{1}(1,0)$, namely $\varphi(f)=\left(\partial / \partial z_{2}\right) f(1,0)$ is discontinuous in the ball norm. Thus the closure in $A\left(\mathbb{B}^{2}\right)$ is the whole algebra.

This argument readily generalizes to $n>2$.

Example 2.8. It is easy to see that if $\Omega$ is contained in a large ball which is tangent to $\Omega$ at a point $\alpha$, then $\Omega$ is pointed there. So any convex domain which has strictly positive curvature at each point on the boundary will be a pointed domain. 


\section{The commutator ideal}

We now consider the nature of the range of the Generalized Berezin Transform. Our goal is to establish that the quotient of $\mathfrak{T}(\mathscr{R})$ by its commutator ideal is large, and our tool for establishing that $\mathfrak{T}(\mathscr{R})$ has many characters is the GBT.

By Proposition 1.5, the map taking $z$ to $\Gamma X(z)=V_{z}^{*} X V_{z}$ is a bounded, continuous $\mathfrak{L}\left(\ell_{m}^{2}\right)$-valued function on $\Omega$ for $X$ in $\mathfrak{L}(\mathscr{R})$. Hence we can extend $\Gamma X$ to the Stone-Čech compactification $\beta \Omega$ of $\Omega$. The inclusion of $A(\Omega)$ in $C(\beta \Omega)$ defines a map $\rho: \beta \Omega \rightarrow M_{A}$. Suppose for convenience that $m=1$. For $\alpha$ in $\beta \Omega$, we can ask if the state on $\mathfrak{T}(\mathscr{R})$ defined by $X \rightarrow \Gamma(X)(\alpha)$ is multiplicative. If it is, then it must coincide with the extension of the map on $\mathfrak{T}(\mathscr{R})$ defined by $M_{\varphi} \rightarrow \widehat{\varphi}(\rho(\alpha))$. Thus there is a close relationship between the Berezin transform on $\mathfrak{T}(\mathscr{R})$ and the Gelfand transform on $\mathfrak{T}(\mathscr{R}) / \mathfrak{C}(\mathscr{R})$, which we investigate in this section. Our approach is based on the fact that $A(\Omega)$ is pointed.

The following lemma is the key observation that takes advantage of functions which attain their maximum modulus at $\alpha$. Let $P_{z}=V_{z} V_{z}^{*}$ be the projection onto the range of $V_{z}$ for $z \in \Omega$.

Lemma 3.1. Suppose that $\alpha \in M_{A}$ and $\varphi$ is a function in $A(\Omega)$ such that $|\widehat{\varphi}(\alpha)|=$ $\|\varphi\|_{A(\Omega)}$. Then $\lim _{z \rightarrow \alpha}\left\|\left[P_{z}, M_{\varphi}\right]\right\|=0$. That is, the range of $V_{z}$ is asymptotically reducing for $M_{\varphi}$ as $\boldsymbol{z}$ approaches $\alpha$.

For any $\psi \in A(\Omega)$ and $S \in \mathfrak{L}(\mathscr{R})$, it follows that

$$
\lim _{z \rightarrow \alpha}\left\|\Gamma\left(S\left[M_{\psi}^{*}, M_{\varphi}\right]\right)(z)\right\|_{\mathfrak{L}\left(\ell_{m}^{2}\right)}=0 .
$$

Proof. Assume that $\|\varphi\|=1$. Decompose $\mathscr{R}=\left(\right.$ range $\left.V_{z}\right) \oplus\left(\text { range } V_{z}\right)^{\perp}$, and write $M_{\varphi}$ as a matrix relative to the decomposition. Since $V_{z}^{*} M_{\varphi}=\varphi(z) V_{z}^{*}$, we obtain

$$
M_{\varphi} \simeq\left[\begin{array}{cc}
\varphi(z) I_{\text {range }} V_{z} & 0 \\
C_{z} & D_{z}
\end{array}\right] \text { and } P_{z}=\left[\begin{array}{cc}
I_{\text {range }} V_{z} & 0 \\
0 & 0
\end{array}\right] .
$$

Moreover, since

$$
\left\|M_{\varphi}\right\|_{\mathfrak{L}(\mathscr{R})} \leq\|\varphi\|_{A(\Omega)}=1 \quad \text { and } \quad|\varphi(\boldsymbol{z})| \leq\|\varphi\|_{A(\Omega)}=1,
$$

it follows that $C_{z}=\left(1-|\varphi(z)|^{2}\right)^{1 / 2} C_{z}^{\prime}$, where $\left\|C_{z}^{\prime}\right\| \leq 1$, whence we obtain that $\left\|C_{\boldsymbol{z}}\right\| \leq\left(1-|\varphi(\boldsymbol{z})|^{2}\right)^{1 / 2}$. Therefore

$$
\left\|\left[M_{\varphi}, P_{z}\right]\right\|=\left\|\left[\begin{array}{cc}
0 & 0 \\
C_{z} & 0
\end{array}\right]\right\| \leq\left(1-|\varphi(\boldsymbol{z})|^{2}\right)^{1 / 2} .
$$

Hence

$$
\lim _{z \rightarrow \alpha}\left\|\left[M_{\varphi}, P_{z}\right]\right\| \leq \limsup _{z \rightarrow \alpha}\left(1-|\varphi(z)|^{2}\right)^{1 / 2}=\left(1-|\hat{\varphi}(\alpha)|^{2}\right)^{1 / 2}=0 .
$$


Write

$$
M_{\psi}^{*} \simeq\left[\begin{array}{cc}
\overline{\psi(z)} I_{\text {range }} V_{z} & X_{z} \\
0 & Y_{z}
\end{array}\right] \text { and } S=\left[\begin{array}{ll}
S_{11} & S_{12} \\
S_{21} & S_{22}
\end{array}\right]
$$

A straightforward calculation yields the $(1,1)$ entry of the operator $S\left[M_{\psi}^{*}, M_{\varphi}\right]$ :

$$
\Gamma\left(S\left[M_{\psi}^{*}, M_{\varphi}\right]\right)(z)=V_{z}^{*}\left(S_{11} X_{z}+S_{12}\left(Y_{z}-\bar{\psi}(z)\right) C_{z} V_{z}\right.
$$

This is easily bounded by $3\|\psi\|\|S\|\left\|C_{z}\right\|$ which tends to 0 as $z$ converges to $\alpha$.

Theorem 3.2. If $\Omega$ is pointed at $\alpha \in M_{A}$, then

$$
\lim _{z \rightarrow \alpha} \Gamma(T)(z)
$$

exists for all $T$ in $\mathfrak{T}(\Re)$, and this map defines a character of $\mathfrak{T}(\mathscr{R})$ corresponding to evaluation at $\alpha$. In particular, the GBT evaluated at $\alpha$ in this way annihilates the commutator ideal of $\mathfrak{T}(\mathscr{R})$.

Proof. By Lemma 3.1, the ranges of $V_{z}$ are asymptotically reducing for operators $M_{\varphi}$ corresponding to those functions $\varphi$ in $A(\Omega)$ which peak at $\alpha$. By hypothesis, these functions generate $A(\Omega)$. However it is a straightforward calculation to see that the set of operators $T \in \mathfrak{L}(\mathscr{R})$ with the property that $\lim _{z \rightarrow \alpha}\left\|\left[T, P_{z}\right]\right\|=0$ is a $\mathrm{C}^{*}$-algebra. Consequently it contains all of $\mathfrak{T}(\mathscr{R})$.

The limit

$$
\lim _{z \rightarrow \alpha} \Gamma\left(M_{\varphi}\right)(\boldsymbol{z})=\varphi(\alpha) I_{\mathfrak{L}\left(\ell_{m}^{2}\right)}
$$

exists for all $\varphi \in A(\Omega)$. Moreover, it follows that compression to the ranges of $V_{z}$ is asymptotically multiplicative. Therefore, this limit exists for all $T \in \mathfrak{T}(\mathscr{R})$, and the map is a $*$-homomorphism.

As the generators are sent to a family of scalar operators, the image consists of scalars. Hence this determines a (unique) character extending evaluation at the point $\alpha$. In particular, this map annihilates the commutator ideal.

Arguments such as those given above have been used in studying the Berezin transform on the Hardy and Bergman modules for $\mathbb{D}$ (see [Hedenmalm et al. 2000; McDonald et al. 1979; Axler and Zheng 2004]) but the role of pointedness was only implicit. A somewhat related notion appears in [Arveson 1969, Theorem 2.2.7], where one assumes that the collection of unitary operators in the closed span of

$$
\left\{M_{\varphi}+M_{\psi}^{*}: \varphi, \psi \in A(\Omega)\right\}
$$

generates $\mathfrak{T}(\mathscr{R})$. Our results hold without special hypotheses.

Theorem 3.3. Let $\Omega$ be a domain in $\mathbb{C}^{n}$ and let $\mathscr{R}$ be a finite-rank quasi-free Hilbert module over $A(\Omega)$. Then $\bar{\Gamma}=\left.\Gamma\right|_{\mathfrak{T}(\mathscr{R})}$ extends to a $*$-homomorphism from $\mathfrak{T}(\mathscr{R})$ to $C(\partial A)$. The null space of $\bar{\Gamma}$, denoted $\gamma(\mathfrak{T}(\mathscr{R}))$ and called the Berezin nullity, is an ideal which contains $\mathfrak{C}(\mathscr{R})$. 
Proof. Izzo's result (Theorem 2.3) shows that $A(\Omega)$ is pointed. Hence, by Theorem 3.2 ,

$$
\bar{\Gamma}(T)(\alpha):=\lim _{z \rightarrow \alpha} \Gamma(T)(z)
$$

is defined at all p-points in the Šilov boundary. That is, evaluation at $\alpha$ extends to a character on the $\mathrm{C}^{*}$-algebra $\mathfrak{T}(\mathscr{R})$.

The set of characters on $\mathfrak{T}(\mathscr{R})$ is a compact set $X_{\mathscr{R}}$. Restriction to $A(\Omega)$ yields a map of $X_{\mathscr{R}}$ into $M_{A}$. This map is injective because $\left\{M_{\varphi}: \varphi \in A(\Omega)\right\}$ generated $\mathfrak{T}(\mathscr{R})$. From the previous paragraph, $X_{\mathscr{R}}$ contains all p-points, and hence contains their closure, which is the Šilov boundary.

It follows that $\bar{\Gamma}(T)$ extends by continuity on $\partial A$ to all points of the Šilov boundary, yielding a $*$-homomorphism of $\mathfrak{T}(\mathscr{R})$ into $C(\partial A)$. Since $A(\Omega)$ separates points in $M_{A}$ and thus in $\partial A$, this map is surjective by the Stone-Weierstrass theorem.

In many situations which occur in practice, every point in the topological boundary of $\Omega$ is a peak point. In this case, a much stronger conclusion is possible.

Corollary 3.4. Assume that the Šlov boundary $\partial A$ of $A(\Omega)$ coincides with the topological boundary $\partial \Omega$, and furthermore that every point in $\partial A$ is a peak point. Then the generalized Berezin transform $\Gamma(T)$ is uniformly continuous on $\Omega$ and extends to $\bar{\Omega}$ and takes scalar values on the boundary.

If every point of $\partial A$ is a peak point but $\partial A$ does not coincide with the topological boundary $\partial \Omega$, then the GBT extends to be continuous on $\Omega \cup \partial A$.

Proof. It is routine to verify that once we know (from Theorem 3.3) that $\Gamma(T)(z)$ extends by continuity to every point on $\partial \Omega$, that $\Gamma(T)$ is continuous on $\bar{\Omega}$. Moreover since evaluation at each boundary point is a character, it takes scalar values there.

Likewise, if we only know continuity of the GBT at each point of $\partial A$, then we at least obtain continuity on $\Omega \cup \partial A$. But as we then have no information about the GBT as it approaches boundary points which are not in the Šilov boundary, we cannot make any claim to uniform continuity.

Remark 3.5. We have not been able to determine whether or not the GBT extends by continuity to the Šilov boundary when there are nonpeak points. The well known examples all fall within the purview of the previous corollary, and so do not provide any insight into this question.

What we do obtain in general is the fact that the character space always contains the Šilov boundary.

Corollary 3.6. Let $\Omega$ be a domain in $\mathbb{C}^{n}$ and let $\mathscr{R}$ be a finite-rank quasi-free Hilbert module over $A(\Omega)$. Then $\partial A \subseteq X_{\mathscr{R}} \subseteq M_{A(\Omega)}$. 
Proof. Consider the following diagram (whose bottom row is not exact):

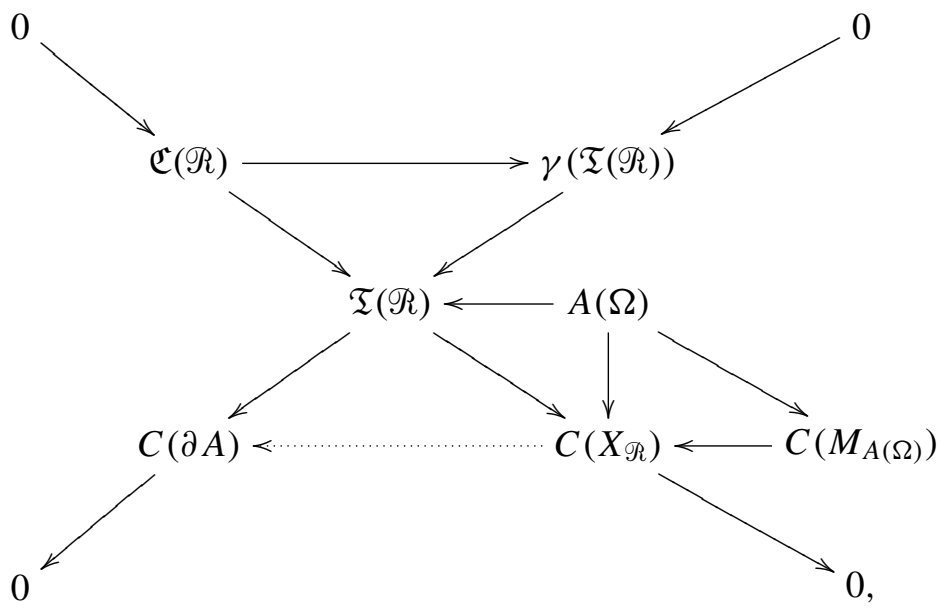

We see that the map of $\mathfrak{C}(\mathscr{R})$ into $\gamma(\mathfrak{T}(\mathscr{R}))$, which is defined by inclusion, yields the dotted map from $C\left(X_{\mathscr{R}}\right)$ to $C(\partial A)$. The inclusion $\partial A \subseteq X_{\mathscr{R}}$ follows.

Recall that we have $X_{\mathscr{R}}=\partial A$ for $\mathscr{R}$ the Hardy or Bergman module but this equality does not hold in general. This matter comes down to the possible equality of $\mathfrak{C}(\mathscr{R})$ with $\gamma(\mathfrak{T}(\mathscr{R}))$.

Corollary 3.7. Let $\Omega$ be a bounded domain in $\mathbb{C}^{n}$. Let $\left\{\varphi_{i}\right\}$ and $\left\{\psi_{i}\right\}$ be sets of functions in $A(\Omega)$, and let $C \in \mathfrak{C}(\mathscr{R})$. It follows that

$$
\left\|\sum_{i} M_{\varphi_{i}} M_{\psi_{i}}^{*}+C\right\|_{\mathcal{L}(\Re)} \geq \sup _{\alpha \in \partial A}\left|\sum \widehat{\varphi}_{i}(\alpha) \overline{\widehat{\psi}_{i}(\alpha)}\right| .
$$

Actually the inequality holds for $C$ in $\gamma(\mathfrak{T}(\mathscr{R}))$. Observe that $\mathfrak{T}(\mathscr{R})$, the closed span of the set

$$
\left\{M_{\varphi} M_{\psi}^{*}+\mathfrak{C}(\mathscr{R}): \varphi, \psi \in A(\Omega)\right\},
$$

is closed under multiplication. This follows from the identity

$$
\begin{aligned}
M_{\varphi_{1}} M_{\psi_{1}}^{*} M_{\varphi_{2}} M_{\psi_{2}}^{*} & =M_{\varphi_{1} \varphi_{2}} M_{\psi_{1} \psi_{2}}^{*}+M_{\varphi_{1}}\left(M_{\psi_{1}}^{*} M_{\varphi_{2}}-M_{\varphi_{2}} M_{\psi_{1}}^{*}\right) M_{\psi_{2}}^{*} \\
& =M_{\varphi_{1} \varphi_{2}} M_{\psi_{1} \psi_{2}}^{*}+M_{\varphi_{1}}\left[M_{\psi_{1}}^{*}, M_{\varphi_{2}}\right] M_{\psi_{2}}^{*}
\end{aligned}
$$

since the second operator is in $\mathfrak{C}(\mathscr{R})$. So this corollary is a statement about all elements of $\mathfrak{T}(\mathscr{R})$.

Definition 3.8. Let the Berezin spectrum $\sigma_{B}(\mathscr{R})$ of $\mathscr{R}$ denote the set of all points $\alpha$ in $M_{A}$ such that $\lim _{z \rightarrow \alpha} \Gamma(T)(z)$ exists for all $T \in \mathfrak{T}(\mathscr{R})$.

Theorem 3.2 shows that $\sigma_{B}(\mathscr{R})$ contains all p-points, and thus is dense in the Šilov boundary $\partial A$ of $A(\Omega)$. The following result shows that it is always a subset of the topological boundary of $\Omega$. 
Lemma 3.9. A quasi-free module $\mathscr{R}$ over $A(\Omega)$ has no reducing eigenvectors.

Proof. Suppose that $x$ is a reducing eigenvector for $\mathscr{R}$. Then the functional $\Phi(A)=$ $(A x, x)$ is multiplicative on the $\mathrm{C}^{*}$-algebra $\mathfrak{T}(\mathscr{R})$, and thus determines a point $\xi$ in the character space $X_{\mathscr{R}}$.

Suppose that $z \in \Omega$ and $z \neq \xi$. We claim that range $V_{z}$ is orthogonal to $x$. Indeed if $k=\alpha x+y$, with $y$ orthogonal to $x$, lies in range $V_{z}$, then for any $\varphi \in A(\Omega)$,

$$
\overline{\operatorname{eval}_{z}(\varphi)} \alpha x+\overline{\operatorname{eval}_{z}(\varphi)} y=\overline{\varphi(z)} k=M_{\varphi}^{*} k=\overline{\Phi(\varphi)} \alpha x+M_{\varphi}^{*} y .
$$

Moreover $M_{\varphi}^{*} y$ is orthogonal to $x$, and so if $\alpha \neq 0$, $\operatorname{eval}_{z}(\varphi)=\Phi(\varphi)$. Hence $\Phi=\operatorname{eval}_{z}$ rather than evaluation at $\xi$. This contradiction establishes the claim.

However, the span of the ranges of $V_{z}$ for $z \neq \xi$ is dense in $\mathscr{H}$. Therefore there are no reducing eigenvalues.

Corollary 3.10. The Berezin spectrum of $\Re$ is contained in the topological boundary of $\Omega$.

Proof. The Berezin spectrum is contained in the closure of $\Omega$ in the maximal ideal space $M_{A}$, which is $\Omega \cup \partial \Omega$. However at a point $z_{0}$ interior to $\Omega$, the limit is actually the GBT evaluated at $z_{0}$. For this to be multiplicative on $\mathfrak{T}(\mathscr{R})$, the range of $V_{z_{0}}$ must reduce $\mathfrak{T}(\mathscr{R})$. The generators $M_{\varphi}$ for $\varphi$ in $A(\Omega)$ are sent to the scalar operators $\varphi\left(z_{0}\right) I_{\mathfrak{L}\left(\ell_{m}^{2}\right)}$, and thus all of $\mathfrak{T}(\mathscr{R})$ is mapped into the scalars. Therefore the range of $V_{z_{0}}$ must consist of reducing eigenvalues. This contradicts Lemma 3.9 , and thus the Berezin spectrum is contained in the boundary of $\Omega$.

\section{Examples}

In this section, we present a variety of examples exploring the possibilities for the character space and the GBT.

The following result shows the implication of Theorem 3.3 in the one variable case in the presence of other hypotheses on $\mathscr{R}$.

Theorem 4.1. Let $\Omega$ be a bounded domain in $\mathbb{C}$ and $\mathscr{R}$ be a quasi-free Hilbert module over $A(\Omega)$, of finite rank $m$.

(1) For $z_{0}$ in $\Omega$, range $\left(M_{z-z_{0}}\right)$ is closed if and only if $M_{z-z_{0}}$ is a Fredholm operator of index $-m$.

(2) If range $\left(M_{z-z_{0}}\right)$ is closed for $z_{0}$ in $\Omega$ and $m=1$ or $\mathfrak{T}(\mathscr{R})$ is irreducible, then $\mathfrak{K}(\mathscr{R}) \subseteq \mathfrak{C}(\mathscr{R}) \subseteq \gamma(\mathfrak{T}(\mathscr{R}))$.

(3) If range $\left(M_{z-z_{0}}\right)$ is closed for all $z_{0}$ in $\Omega$, then $\mathfrak{T}(\mathscr{R}) / \mathfrak{K}(\mathscr{R})=C(\partial A)$, which implies $\mathfrak{K}(\mathscr{R})=\mathfrak{C}(\mathscr{R})=\gamma(\mathfrak{T}(\mathscr{R}))$. 
Proof. Statement (1) follows since the null space of any module multiplier is (0) and the dimension of the null space of $\left(M_{z-z_{0}}\right)^{*}$ is $m$. Statement (2) follows from the fact that a $\mathrm{C}^{*}$-algebra containing a Fredholm operator of nonzero index must contain a nonzero compact operator and the fact that the set of compact operators is the minimal ideal in any $\mathrm{C}^{*}$-algebra containing it. Statement (3) follows from the well known fact that a Fredholm operator in a $\mathrm{C}^{*}$-algebra containing the compact operators is invertible modulo the ideal of compact operators.

It is not always true in the context above that $\mathfrak{C}=\mathfrak{K}$.

Example 4.2. Let $\ell^{2}$ be the Hilbert space with orthonormal basis $\left\{e_{k}\right\}_{k \in \mathbb{N}}$, and let $\Sigma$ be an infinite subset of $\mathbb{N}$ for which $\Sigma \cap(\Sigma+k)$ is finite for all $k \geq 1$ and

$$
\lim _{n \rightarrow \infty} \frac{1}{n} \operatorname{card}\{k \in \Sigma: 0 \leq k<n\}=0 .
$$

Let $P_{\Sigma}$ be the projection in $\mathfrak{L}\left(\ell^{2}\right)$ onto the span of $\left\{e_{k}: k \in \Sigma\right\}$ and $S$ be the unilateral shift on $\ell^{2}$, that is, $S e_{k}=e_{k+1}, k \geq 1$. Let $A$ be the weighted shift $A=S\left(I-\frac{1}{2} P_{\Sigma}\right)$. Then the point spectrum of $A^{*}$ is $\mathbb{D}$ and hence $A$ defines a rankone quasi-free Hilbert module over $A(\mathbb{D})$. By considering the polar decomposition of $A$, we see that the $\mathrm{C}^{*}$-algebra $\mathfrak{A}$ generated by $I$ and $A$ is equal to the $\mathrm{C}^{*}$-algebra generated by $S$ and $P_{\Sigma}$. Moreover, $\mathfrak{A}$ contains $\mathfrak{K}=\mathfrak{K}\left(\ell^{2}\right)$.

Let $\mathscr{B}=\mathfrak{A} / \mathfrak{K}$ and $u$ and $p$ be the images of $S$ and $P_{\Sigma}$ in $\mathscr{B}$, respectively. Then $u$ is unitary and $p$ is a projection with the property that the projections $\left\{u^{* k} p u^{k}\right\}_{k \in \mathbb{Z}}$ are pairwise orthogonal. Hence, the ideal $\mathscr{F}$ in $\mathscr{B}$ generated by $p$ is isomorphic to $\mathfrak{K}$. Moreover, $\mathscr{B}$ is a trivial extension of $\mathfrak{K}$ by $C(\partial \mathbb{D})$, that is, $\mathscr{B} / \mathfrak{K} \simeq C(\partial \mathbb{D})$ and the map $z \rightarrow u$ extends to an isomorphism from $C(\partial \mathbb{D})$ to $\mathscr{B}$.

Now the commutator ideal $\mathfrak{C}$ of $\mathfrak{A}$ contains $\mathfrak{K}$ and is the preimage of $\mathscr{g}$ in $\mathfrak{A}$. Therefore, $\mathfrak{A} / \mathfrak{C} \simeq C(\partial \mathbb{D})$ but $\mathfrak{A} / \mathfrak{K}=\mathscr{B}$ is not commutative. Hence, $\mathfrak{K} \neq \mathfrak{C}$ in this case. However, $\mathfrak{C}$ and the Berezin nullity are equal.

There is another hypothesis that would imply the coincidence of $\gamma(\mathfrak{T}(\mathscr{R}))$ and $\mathfrak{C}(\mathscr{R})$ in the context of Theorem 4.1. If none of the points in $\Omega$ lie in $X_{\mathscr{R}}$, then $X_{\mathscr{R}}$ must be a subset of $\partial \Omega$. Since $\partial \Omega \subset X_{\mathscr{R}}$, we have $X_{\mathscr{R}}=\partial \Omega$ which is the maximal ideal space of $\mathfrak{T}(\mathscr{R}) / \gamma(\mathfrak{T}(\mathscr{R}))$. Hence, $\mathfrak{C}(\mathscr{R})=\gamma(\mathfrak{T}(\mathscr{R}))$. Note that this latter argument is valid for the case of a domain $\Omega$ in $\mathbb{C}^{n}$ if $\Omega$ is dense in $M_{A(\Omega)}$ and $\partial A=\partial \Omega$.

The extension of the preceding results to the several variables context would be of particular interest. One approach would be to assume that the last stage of the Koszul complex, which defines the Taylor spectrum, has closed range. In this way, one can show that $X_{\mathscr{R}}=\partial \mathbb{B}^{n}$ for $\mathscr{R}$ equal to $H^{2}\left(\mathbb{B}^{n}\right)$ or $B^{2}\left(\mathbb{B}^{n}\right)$ and $X_{H^{2}\left(\mathbb{D}^{n}\right)}=\mathbb{T}^{n}$ for all $n$. We will not provide the details.

Now we consider when a point of the maximal ideal space lies in $X_{\mathscr{R}}$. We shall see that $X_{\Re}$ can be a fairly general subset containing the Šilov boundary. Our 
argument works for any finitely generated algebra. Our main application, Corollary 4.4, is a strengthening of an old result of Bunce [1971] where hyponormality is assumed. This paper was one of the precursors of Voiculescu's celebrated Weylvon Neumann theorem [Voiculescu 1976] (see [Davidson 1996, Lemma II.5.5]) which we use below.

Theorem 4.3. Let $A$ be a unital operator algebra represented on a Hilbert space $\mathscr{H}$, and $A_{1}, \ldots, A_{n}$ be a finite set of generators for $\mathscr{A} ;$ and let $\mathfrak{A}=C^{*}(\mathscr{A})$. A multiplicative functional $\Phi$ on $\mathscr{A}$ extends to a character of $\mathfrak{A}$ if and only if the column operator $T$ with entries $A_{i}-\Phi\left(A_{i}\right) I$ and $A_{i}^{*}-\overline{\Phi\left(A_{i}\right)}$ for $1 \leq i \leq n$ is not bounded below.

Proof. Suppose that $\Phi$ extends to a character $\bar{\Phi}$ of $\mathfrak{A}$. If $\bar{\Phi}$ is nonzero on the ideal $\mathfrak{A} \cap \mathfrak{K}$ of compact operators, it must correspond to a finite dimensional reducing subspace $\mathcal{M}$ on which $\left.T_{i}\right|_{\mathcal{M}}=\Phi\left(T_{i}\right) I_{\mathcal{M}}$. Hence the column operator $T$ has $\mathcal{M}$ in its kernel. Otherwise $\bar{\Phi}$ annihilates $\mathfrak{A} \cap \mathfrak{K}$. So by Voiculescu's Theorem, the identity representation absorbs countably many copies of $\bar{\Phi}$, that is id $\sim_{a} \operatorname{id} \oplus \bar{\Phi}^{(\infty)}$. This means that there is an orthonormal sequence $\left\{x_{k}\right\}$ of vectors which are asymptotically reducing and

$$
\lim _{k \rightarrow \infty}\left\|A_{i} x_{k}-\Phi\left(A_{i}\right) x_{k}\right\|=0=\lim _{k \rightarrow \infty}\left\|A_{i}^{*} x_{k}-\overline{\Phi\left(A_{i}\right)} x_{k}\right\| .
$$

So $\lim _{k \rightarrow \infty}\left\|T x_{k}\right\|=0$.

Conversely, suppose that $T$ is not bounded below. This could occur because there is a unit vector $x$ in its kernel. Then

$$
A_{i} x=\Phi\left(A_{i}\right) x \quad \text { and } \quad A_{i}^{*} x=\overline{\Phi\left(A_{i}\right)} x .
$$

So $x$ is a reducing eigenvalue for the operators $A_{1}, \ldots, A_{n}$, and hence for the $\mathrm{C}^{*}$ algebra $\mathfrak{A}$. Clearly restriction to $\mathbb{C} x$ is a $*$-homomorphism, and thus determines a character $\bar{\Phi}$ of $\mathfrak{A}$ which extends $\Phi$.

On the other hand, if $T$ is injective, there must be an orthonormal sequence $\left\{x_{k}: k \geq 1\right\}$ so that $\lim _{k \rightarrow \infty}\left\|T x_{k}\right\|=0$. Let $P_{k}=x_{k} x_{k}^{*}$ denote the rank-one projection onto $\mathbb{C} x_{k}$. Then

$$
\begin{aligned}
\left\|\left[A_{i}, P_{k}\right]\right\| & =\max \left\{\left\|P_{k}^{\perp} A_{i} P_{k}\right\|,\left\|P_{k} A_{i} P_{k}^{\perp}\right\|\right\} \\
& \leq \max \left\{\left\|\left(A_{i}-\Phi\left(A_{i}\right)\right) x_{k}\right\|,\left\|\left(A_{i}-\Phi\left(A_{i}\right)\right)^{*} x_{k}\right\|\right\}
\end{aligned}
$$

Hence $\lim _{k \rightarrow \infty}\left\|\left[A_{i}, P_{k}\right]\right\|=0$. It follows that $\lim _{k \rightarrow \infty}\left\|\left[A, P_{k}\right]\right\|=0$ for every $A \in \mathfrak{A}$. In particular,

$$
\lim _{k \rightarrow \infty}\left(A_{i} x_{k}, x_{k}\right)=\Phi\left(A_{i}\right) \quad \text { and } \quad \lim _{k \rightarrow \infty}\left(A_{i}^{*} x_{k}, x_{k}\right)=\overline{\Phi\left(A_{i}\right)} .
$$


Define an extension $\bar{\Phi}(A)=\lim _{k \rightarrow \infty}\left(A x_{k}, x_{k}\right)$. It is clear that this limit exists for any polynomial in the generators and their adjoints, and hence, by continuity, for the whole $\mathrm{C}^{*}$-algebra. Then for $A, B \in \mathfrak{A}$,

$$
\begin{aligned}
& \left|\left(B A x_{k}, x_{k}\right)-\left(A x_{k}, x_{k}\right)\left(B x_{k}, x_{k}\right)\right| \\
& =\left|\left(P_{k} A x_{k}, P_{k} B^{*} x_{k}\right)+\left(P_{k}^{\perp} A x_{k}, P_{k}^{\perp} B^{*} x_{k}\right)-\left(P_{k} A x_{k}, x_{k}\right)\left(x_{k}, P_{k} B^{*} x_{k}\right)\right| \\
& =\left|\left(P_{k}^{\perp} A x_{k}, P_{k}^{\perp} B^{*} x_{k}\right)\right| \leq\left\|P_{k}^{\perp} A P_{k}\right\|\left\|P_{k}^{\perp} B^{*} P_{k}\right\| .
\end{aligned}
$$

Hence $\bar{\Phi}(B A)=\bar{\Phi}(A) \bar{\Phi}(B)$ is multiplicative. So $\bar{\Phi}$ is a character of $\mathfrak{A}$ which extends $\Phi$.

By the previous theorem and Lemma 3.9, we obtain:

Corollary 4.4. Let $\mathscr{R}$ be a quasi-free module over $A(\Omega)$ where $\Omega$ is a domain in $\mathbb{C}^{n}$. Then evaluation at a point $\omega \in \Omega$ extends to a multiplicative functional on $\mathfrak{T}(\mathscr{R})$ if and only if the column operator $T$ with entries $M_{z_{i}-\omega_{i}}$ and $M_{z_{i}-\omega_{i}}^{*}$ for $1 \leq i \leq n$ is not bounded below.

Example 4.5. Consider a contractive rank-one module over $A(\mathbb{D})$ which is rotation invariant in the sense that sending $z$ to $\lambda z$, where $|\lambda|=1$, induces an isometric automorphism of $\mathscr{R}$. It is routine to verify that this implies that $\left\{z^{k}: k \geq 0\right\}$ is an orthogonal set spanning $\mathscr{R}$. Hence $M_{z}$ is an injective weighted shift. Being a representation of $A(\mathbb{D})$, we see that $\left\|M_{z}\right\|=1=\operatorname{spr}\left(M_{z}\right)$ (where $\operatorname{spr}(T)$ is the spectral radius of $T$ ). All weighted shifts are unitarily equivalent to a shift with positive weights, so we may suppose that $M_{z} \simeq T$ where $T$ acts on $\ell^{2}$ by $T e_{n}=a_{n} e_{n}$ and $0<a_{n} \leq 1$ for $n \geq 0$.

The problem of determining the set $X_{\Re}$ for this module comes down to determining when the column operator with entries $T-\lambda I$ and $(T-\lambda I)^{*}$ is bounded below. Equivalently, we may consider the operator

$$
X=(T-\lambda I)^{*}(T-\lambda I)+(T-\lambda I)(T-\lambda I)^{*} .
$$

Suppose that for each $\varepsilon>0$ and $d \in \mathbb{N}$, there is an integer $k$ so that

$$
\left|a_{k+i}-\right| \lambda||<\varepsilon \text { for } 0 \leq i \leq d+1,
$$

then with $\lambda=e^{i \theta}|\lambda|$, we may set $x_{k}=d^{-1 / 2} \sum_{j=1}^{d} e^{-i j \theta} e_{k+i}$. It is a simple exercise to show that $\left\|(T-\lambda I) x_{k}\right\|$ and $\left\|(T-\lambda I)^{*} x_{k}\right\|$ are both small. In the limit, we see that the column operator is not bounded below and $\lambda$ lies in $X_{\mathscr{R}}$.

On the other hand, if $a_{k}$ is bounded away from $|\lambda|$ for all $k \geq 0$, say by $\delta>0$, then $X$ is invertible. Indeed, one can easily see that

$$
X=T^{*} T+T T^{*}+2|\lambda|^{2} I-2\left(\bar{\lambda} T+\lambda T^{*}\right) .
$$


This is a tridiagonal operator with entries

$$
x_{i i}=a_{i-1}^{2}+a_{i}^{2}+2|\lambda|^{2} \quad x_{i, i+1}=2 \bar{\lambda} a_{i} \quad \text { and } \quad x_{i+1, i}=2 \lambda a_{i} .
$$

(We set $a_{-1}=0$.) Then $X$ is the sum of two operators,

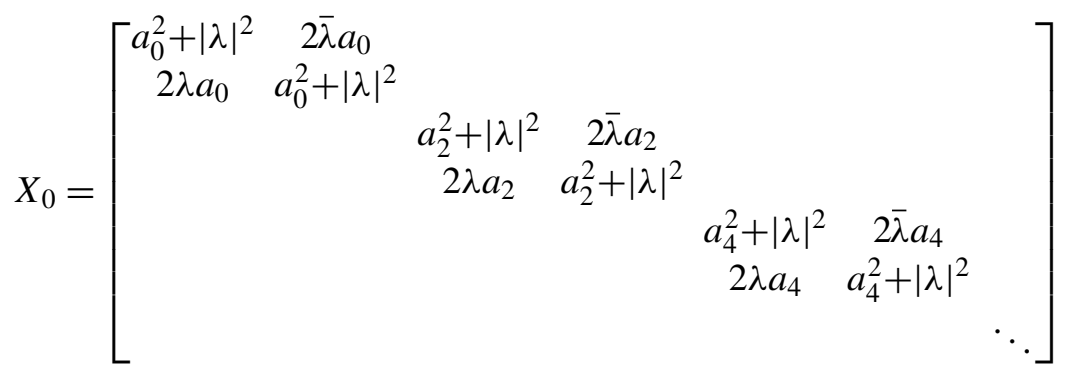

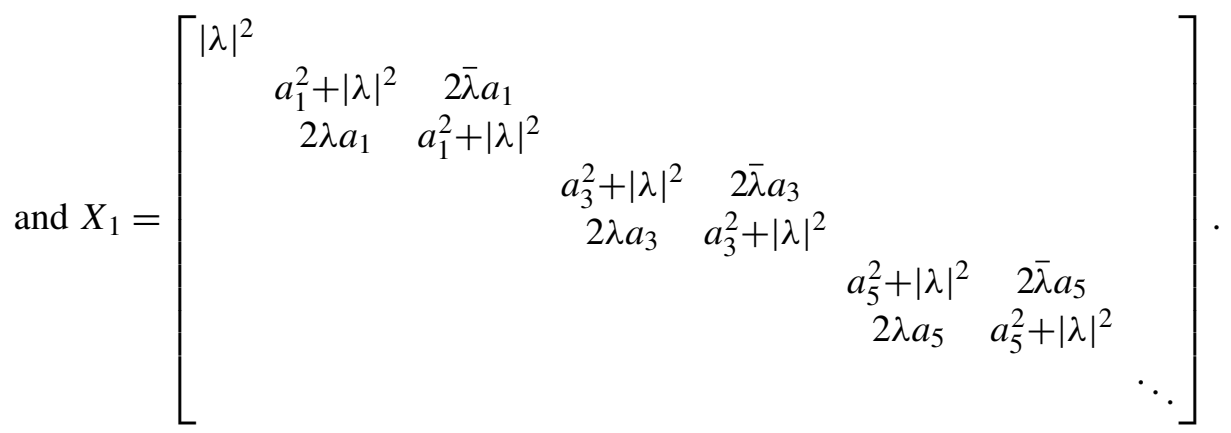

It is now easy to check that $X \geq X_{0} \geq \delta^{2} / 2 I$. So $\lambda$ does not lie in $X_{\mathscr{R}}$.

A similar analysis, which we omit, shows that if there is a $\delta>0$ and an integer $d$ so that there are no strings of weights of length $d$ all within $\delta$ of $|\lambda|$, then again $X$ is bounded below; and so $\lambda$ does not lie in $X_{\mathscr{R}}$. Various spectral properties of weighted shifts have been studied in detail. See Shields [1974] for an overview. This result could well be known, but we have been unable to find it in the literature.

Let $C$ be a compact subset of $[0,1]$ containing 1 in which 0 is not an isolated point. Choose a sequence $\left\{r_{k}: k \geq 1\right\}$ contained in $C \backslash\{0\}$ so that every point in $C$ is a cluster point. Then define $T$ to be the weighted shift with weights

$$
r_{1}, r_{2}, r_{2}, r_{3}, r_{3}, r_{3}, r_{4}, r_{4}, r_{4}, r_{4}, \ldots
$$

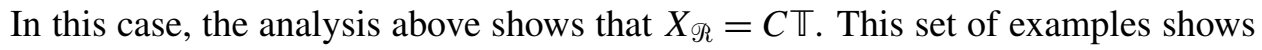
that $X_{\mathscr{R}}$ can be a rather arbitrary subset of $M_{A}$ containing $\partial M_{A}$.

For another example, if $0<r<1$, let the weights $a_{n}$ slowly oscillate back and forth between 1 and $r$. That is, $\left|a_{i}-a_{i+1}\right|$ tends to 0 . Then $T$ is essentially normal with essential spectrum equal to the annulus $\{\lambda: r \leq|\lambda| \leq 1\}$. For this example, it is clear that every point in the annulus is an approximate reducing eigenvalue because $T$ is approximately unitarily equivalent to $S \oplus N$ where $S$ is the unilateral shift and $N$ is normal and has spectrum equal to the annulus. 
In the next example, we show that the disk algebra has a completely bounded module $\mathscr{R}$ (hence similar to a contractive one) in which the set $X_{\mathscr{R}}$ is empty. Indeed, it will be similar to the classical Hardy module. This should be compared to Corollary 3.6 which implies that a quasi-free (hence contractive) module over the unit disk must contain the whole unit circle in $X_{\mathscr{R}}$.

Example 4.6. Fix $0<r<1$ and consider the weighted shift $T$ with weights

$$
1, r^{1 / 2}, 1, r^{3 / 4}, 1, r^{1 / 2}, 1, r^{7 / 8}, \ldots,
$$

namely $a_{n}=r^{1-\operatorname{gcd}\left(n+1,2^{n}\right)^{-1}}$. Note that $T^{2^{k}}$ shifts by $2^{k}$ with weights $b_{n}$ which are the product of $2^{k}$ successive weights of $T$, of which $2^{k-1}$ are $1,2^{k-2}$ are $r^{1 / 2}, \ldots$, one is $r^{1-2^{1-k}}$ and one lies between $r$ and $r^{1-2^{-k}}$. So there is an integer $p=p(n) \geq k$ so that

$$
\begin{aligned}
2^{-k} \log _{r} b_{n} & =1-\left(\frac{1}{2}(1)+\frac{1}{4}\left(\frac{1}{2}\right)+\frac{1}{8}\left(\frac{1}{4}\right)+\cdots+2^{-k} 2^{1-k}+2^{-k} 2^{-p}\right) \\
& =\frac{1}{3}\left(1+2^{1-2 k}\right)-2^{-k-p} .
\end{aligned}
$$

Therefore these weights satisfy

$$
r^{2^{1-k} / 3}<r^{-2^{k} / 3} b_{n} \leq r^{-2^{-k} / 3} .
$$

In particular, the spectral radius of $T$ is $r^{1 / 3}$.

Define $A=r^{-1 / 3} T$. The previous estimate establishes that

$$
\left\|A^{2^{k}}\right\| \leq r^{-2^{-k} / 3}
$$

Hence it is a routine calculation to see that $A$ is power bounded. Likewise, all powers of $A$ are uniformly bounded below. By [Shields 1974], $A$ is similar to the unilateral shift. Thus $A$ determines a bounded module $\mathscr{R}$ which is similar to the Hardy module. Nevertheless, we shall show that $X_{\mathscr{R}}$ is empty.

It is evident that $C^{*}(A)=C^{*}(T)$ is generated by the unilateral shift $S$ and the diagonal operator $D=\left(T^{*} T\right)^{1 / 2}$, both of which come from the polar decomposition of $T$. The spectrum of $D$ is

$$
\sigma(D)=\left\{r, r^{1-\operatorname{gcd}\left(n+1,2^{n}\right)^{-1}}: n \geq 0\right\}
$$

and the spectral projection for the point $r^{1-\operatorname{gcd}\left(n+1,2^{n}\right)^{-1}}$ is the projection onto

$$
\left\{e_{i}: i+1 \equiv 2^{k}\left(\bmod 2^{k+1}\right)\right\} .
$$

It is clear that conjugating these projections by powers of $S$ yields the projections $E_{k, l}$ onto the subspaces spanned by

$$
\left\{e_{i}: i \equiv l\left(\bmod 2^{k}\right)\right\} \quad \text { for } k \geq 1 \text { and } 0 \leq l<2^{k} .
$$


It is easy to see that this is the $\mathrm{C}^{*}$-algebra generated by all weighted shifts of period $2^{k}$ for all $k \geq 0$. This is an extension of the compact operators by the $2^{\infty}$ Bunce-Deddens algebra [Davidson 1996, Section V.3].

The commutator ideal properly contains the compact operators because $A$ is not essentially normal. As the Bunce-Deddens algebras are simple, the commutator ideal is the whole $\mathrm{C}^{*}$-algebra. In particular, there are no characters. Also, by the analysis of Example 4.5, we can see by a different method that there are no characters.

We can also extend our results to cover spherical contractions. Recall that the Hilbert module $\mathcal{M}$ over $A\left(\mathbb{B}^{n}\right)$ is said to be spherically contractive if

$$
\sum_{i=1}^{n}\left\|M_{z_{i}} f\right\|_{\mathscr{R}}^{2} \leq\|f\|_{\mathscr{R}}^{2}
$$

for all $f$ in $\mathscr{R}$.

Theorem 4.7. If $\Re$ is a finite-rank quasi-free Hilbert module over $A\left(\mathbb{B}^{n}\right)$ which is spherically contractive, then the GBT defines a *-homomorphism from $\mathfrak{T}(\mathscr{R})$ onto $C\left(\partial \mathbb{B}^{n}\right)$ with kernel ideal $\gamma(\mathfrak{T}(\mathscr{R}))$ containing $\mathfrak{C}(\mathscr{R})$.

Proof. The first part of the proof is similar to that of Lemma 3.1 but the operator $M_{\varphi}$ on $\mathscr{R}$ is replaced by a column of such operators. Let

$$
\Phi=\left(\begin{array}{c}
\varphi_{1} \\
\vdots \\
\varphi_{k}
\end{array}\right)
$$

be a $(1 \times k)$-column of functions in $A\left(\mathbb{B}^{n}\right)$, and let $M_{\Phi}$ be the corresponding $(1 \times k)$ column matrix of operators acting from $\mathscr{R}$ to $\mathscr{R}^{(k)}$, the direct sum of $k$ copies of $\mathscr{R}$. An easy calculation shows that

$$
\left\|\sum_{i} M_{\varphi_{i}}^{*} M_{\varphi_{i}}\right\|_{\mathcal{L}(\Re)}=\left\|M_{\Phi}^{*} M_{\Phi}\right\|_{\mathcal{L}(\mathscr{R})}=\left\|M_{\Phi}\right\|^{2}
$$

and

$$
\sup _{z \in \mathbb{B}^{n}}\|\Phi(z)\|_{\mathcal{L}\left(\ell_{m}^{2}, \ell_{m}^{2(k)}\right)}^{2}=\sup _{\boldsymbol{z} \in \mathbb{B}^{n}} \sum_{i}\left|\varphi_{i}(\boldsymbol{z})\right|^{2}
$$

Now for the column matrix operator $M_{Z}$ from $\mathscr{R}$ to $\mathscr{R}^{(n)}$, defined by the column matrix

$$
Z=\left(\begin{array}{c}
z_{1} \\
\vdots \\
z_{n}
\end{array}\right)
$$


formed from the coordinate functions, we have

$$
\left\|M_{Z}\right\| \leq \sup _{z \in \mathbb{B}^{n}} \sum_{i=1}^{n}\left|z_{i}\right|^{2}=1
$$

and hence $M_{Z}$ is a spherical contraction.

Next decompose $\mathscr{R}=\mathscr{P}_{z} \oplus \mathscr{Q}_{z}$ for $z$ in $\mathbb{B}^{n}$, where $\mathscr{P}_{z}=$ range $V_{z}$ and $\mathscr{Q}_{z}$ is the orthogonal complement of $\mathscr{P}_{z}$. Similarly, $\mathscr{R}^{(n)}=\mathscr{P}_{z}^{(n)} \oplus \mathscr{Q}_{z}^{(n)}$. Moreover, if we decompose the matrix for the operator $M_{Z}$ relative to this decomposition, we obtain

$$
M_{Z} \simeq\left[\begin{array}{cc}
A_{z} & 0 \\
B_{z} & C_{z}
\end{array}\right], \quad \text { where } A_{z}=\left(\begin{array}{c}
z_{1} I_{\mathscr{P}_{z}} \\
\vdots \\
z_{n} I_{\mathscr{P}_{z}}
\end{array}\right) .
$$

Since $M_{Z}$ is a contraction, by the theorem of Sz.-Nagy and Foiaş, we have that $B_{z}=\left(I-A_{z}^{*} A_{z}\right)^{1 / 2} B_{z}^{\prime}$, where $B_{z}^{\prime}$ is a contraction. But

$$
\left(I-A_{z}^{*} A_{z}\right)^{1 / 2}=\left(1-\sum\left|z_{i}\right|^{2}\right)^{1 / 2}
$$

which implies that $\varlimsup_{\|z\| \rightarrow 1}\left\|B_{z}\right\|=0$.

This result enables us to establish the conclusion of Theorem 3.2 for functions in the closed algebra generated by $\left\{z_{i}\right\}$ which coincides with $A\left(\mathbb{B}^{n}\right)$ and this concludes the proof.

An example of a Hilbert module for which Theorem 4.7 applies (but not Theorem 3.3) is the noncommutative Hardy space $H_{d}^{2}$ which is a module over $\mathbb{C}[z]$ for which the coordinate functions are contractive but $H_{d}^{2}$ is not a bounded module over $A\left(\mathbb{B}^{n}\right)$.

\section{Extension to a larger algebra}

On the disk $\mathbb{D}$ one knows that $H^{2}(\mathbb{D})$ and $B^{2}(\mathbb{D})$ are modules not only for $A(\mathbb{D})$ but for the larger algebra, $H^{\infty}(\mathbb{D})$, of all bounded holomorphic functions over $\mathbb{D}$. The same thing is true for other quasi-free Hilbert modules. In this section we restrict our attention to the rank-one case, $m=1$, although most of the following developments carry over to the higher-rank case. Many of the techniques are well known and often are part of the folklore.

Let $\Re$ be a rank-one quasi-free Hilbert module over $A(\Omega)$, $\operatorname{Hol}(\Omega)$ be the algebra of all holomorphic functions on $\Omega$, and $H^{\infty}(\Omega)$ be the subalgebra of bounded functions in $\operatorname{Hol}(\Omega)$. A function $\varphi$ in $\operatorname{Hol}(\Omega)$ is said to be a multiplier for $\mathscr{R}$ if $\varphi \mathscr{R} \subset \mathscr{R}$. Let $\operatorname{Mul}(\Omega)$ denote the algebra of all multipliers for $\mathscr{R}$ with norm $\|\varphi\|_{\operatorname{Mul}(\mathscr{R})}=\left\|M_{\varphi}\right\|$, where $M_{\varphi}$ is the operator on $\mathscr{R}$ defined to be multiplication by $\varphi$, which is in $\mathfrak{L}(\mathscr{R})$ by the closed graph theorem. Standard arguments show 
that not only must multipliers be bounded, but that $\left\|M_{\varphi}\right\|_{\mathfrak{L}(\mathscr{R})} \geq\|\varphi\|_{H^{\infty}(\Omega)}$. Hence, $\operatorname{Mul}(\Re)$ is a subalgebra of $H^{\infty}(\Omega)$ which is contractively included. In general, the norms are not equal and $\operatorname{Mul}(\Omega) \neq H^{\infty}(\Omega)$. The following lemma shows that $\operatorname{Mul}(\mathscr{R})$ is closed in a rather weak topology.

A sequence $\left\{\varphi_{i}\right\}$ in $A(\Omega)$ is said to converge in the bounded, pointwise limit (bpwl) topology to a function $\varphi$ in $H^{\infty}(\Omega)$ if

(1) $\sup _{i}\left\|\varphi_{i}\right\|_{A(\Omega)}<\infty$ and

(2) $\lim _{i} \varphi_{i}(\boldsymbol{z})=\varphi(\boldsymbol{z})$ for $\boldsymbol{z}$ in $\Omega$.

Lemma 5.1. Let $\mathscr{R}$ be a quasi-free Hilbert module over $A(\Omega)$. Suppose that a bounded sequence $\varphi_{i} \in \operatorname{Mul}(\mathscr{R})$ converges bpwl to $\varphi$. Then $M_{\varphi_{i}}$ converge in the weak operator topology to $M_{\varphi}$. Thus $\left\{M_{\varphi}: \varphi \in \operatorname{Mul}(\mathscr{R}),\|\varphi\|_{H^{\infty}(\Omega)} \leq 1\right\}$ is weak operator closed.

Proof. Let $\varphi$ be in $H^{\infty}(\Omega)$ and $\left\{\varphi_{i}\right\}$ be a bounded sequence in $\operatorname{Mul}(\mathscr{R})$ which converges to $\varphi$ in the bpwl-topology. Since the sequence of operators $\left\{M_{\varphi_{i}}\right\}$ is uniformly bounded, a subsequence of it converges to some $X$ in $\mathfrak{L}(\mathscr{R})$ in the weak operator topology. For a vector $x$ in $\ell_{m}^{2}$ and $g$ in $\mathscr{R}$, we have

$$
\begin{aligned}
\left\langle\operatorname{eval}_{z}(X g), x\right\rangle_{\ell_{m}^{2}} & =\left\langle X g, V_{z} Q_{z} x\right\rangle_{\mathscr{R}}=\lim _{i}\left\langle M_{\varphi_{i}} g, V_{z} Q_{z} x\right\rangle_{\mathscr{R}} \\
& =\lim _{i}\left\langle g, \overline{\varphi_{i}(z)} V_{z} Q_{z} x\right\rangle=\left\langle g, \overline{\varphi(z)} V_{z} Q_{z} x\right\rangle_{\mathscr{R}}=\left\langle\operatorname{eval}_{z}(\varphi g), x\right\rangle_{\ell_{m}^{2}} .
\end{aligned}
$$

Therefore $X g=\varphi g$; whence $X=M_{\varphi}$ and $\varphi$ is in $\operatorname{Mul}(\mathscr{R})$. Since this was valid for any subsequence which had a limit in the weak operator topology, standard arguments show that the original sequence converges to $M_{\varphi}$ in the weak operator topology.

Conversely, suppose that $\varphi_{i} \in \operatorname{Mul}(\mathscr{R})$ such that $M_{\varphi_{i}}$ converge in the weak operator topology to $X$. Then since $\Gamma_{z}(X)=V_{z}^{*} X V_{z}$ is compressed on both sides to the finite dimensional range of $V_{z}$, it follows that

$$
\Gamma_{z}(X)=\lim _{i \rightarrow \infty} \Gamma_{z}\left(M_{\varphi_{i}}\right)=\lim _{i \rightarrow \infty} \varphi_{i}(z) I_{m} .
$$

Therefore there is a bounded pointwise limit $\varphi$ of the sequence $\varphi_{i}$. So $\varphi \in H^{\infty}(\Omega)$ and is a bpwl-limit of multipliers, whence lies in $\operatorname{Mul}(\mathscr{R})$ by the previous paragraph. Repeating the computation above, it follows that $X=M_{\varphi}$.

An argument along the same lines is given in [Curto and Salinas 1984, Thm. 5.2]. If the inclusion is isometric, namely $\|\varphi\|_{\operatorname{Mul}(\mathscr{R})}=\|\varphi\|_{H^{\infty}(\Omega)}$, then

$$
\left\{M_{\varphi}: \varphi \in \operatorname{Mul}(\mathscr{R}),\|\varphi\|_{H^{\infty}(\Omega)} \leq 1\right\}
$$

is the unit ball of the algebra of multipliers $\left.\left\{M_{\varphi}: \varphi \in \operatorname{Mul}(\mathscr{R})\right)\right\}$. In this case, the Krein-Smulyan Theorem shows that this space is weak-* closed. 
Proposition 5.2. Let $\Omega$ be a domain in $\mathbb{C}^{n}$ for which $A(\Omega)$ is dense in $H^{\infty}(\Omega)$ in the bpwl-topology. Then every quasi-free Hilbert module over $A(\Omega)$ extends to a bounded Hilbert module over $H^{\infty}(\Omega)$.

Proof. By Lemma 5.1 and the hypotheses, $H^{\infty}(\Omega)=\operatorname{Mul}(\Omega)$. Therefore, we can define a module action $H^{\infty}(\Omega) \times \mathscr{R} \rightarrow \mathscr{R}$. By the closed graph theorem, there exists a constant $K>0$ such that $\left\|M_{\varphi}\right\|_{\mathfrak{L}(\Re)} \leq K\|\varphi\|_{H^{\infty}(\Omega)}$ for $\varphi$ in $H^{\infty}(\Omega)$.

It is known that various assumptions on $\Omega$ imply that $A(\Omega)$ is dense in $H^{\infty}(\Omega)$ in the bpwl-topology. We provide one easy example. Recall that a domain $\Omega$ in $\mathbb{C}^{n}$ is said to be starlike relative to a point $z_{0}$ in $\Omega$, if for every point $z$ in $\Omega$ the line segment with endpoints $\boldsymbol{z}_{0}$ and $\boldsymbol{z}$ is contained in $\Omega$. Moreover, $\Omega$ is said to be starlike if it is starlike relative to some point in $\Omega$.

If we want $\mathscr{R}$ to be a contractive Hilbert module over $H^{\infty}(\Omega)$, we need more information about how $A(\Omega)$ is bpwl-dense in $H^{\infty}(\Omega)$. The most straightforward hypothesis is the assumption that the sequence $\left\{\varphi_{i}\right\}$ in $A(\Omega)$ converging in the bpwl-topology to a function $\varphi$ in $H^{\infty}(\Omega)$ can be chosen such that $\left\|\varphi_{i}\right\|_{A(\Omega)} \leq$ $\|\varphi\|_{H^{\infty}(\Omega)}$. We will say that $A(\Omega)$ is strongly bpwl-dense in $H^{\infty}(\Omega)$ in that case.

Lemma 5.3. If $\Omega$ is a starlike domain, then $A(\Omega)$ is strongly bpwl-dense in $H^{\infty}(\Omega)$.

Proof. Let $z_{0}$ be a point in $\Omega$ relative to which it is starlike. With $z_{0}$ as origin and $0 \leq t \leq 1$, let $F_{t}$ be the $t$ dilation of $\mathbb{C}^{n}$; that is, $F_{t}(\boldsymbol{z})=\boldsymbol{z}_{0}+t\left(\boldsymbol{z}-\boldsymbol{z}_{0}\right)$ for $\boldsymbol{z}$ in $\mathbb{C}^{n}$. By the starlike hypothesis, we have $F_{t}(\Omega) \subset \Omega$ for $0<t \leq 1$.

For $\varphi$ in $H^{\infty}(\Omega)$, if $\varphi_{t}=\varphi \circ F_{t}$, then $\left\{\varphi_{1-\frac{1}{k}}\right\}$ is a sequence in $A(\Omega)$ for which

$$
\left\|\varphi_{1-\frac{1}{k}}\right\|_{H^{\infty}(\Omega)} \leq\|\varphi\|_{H^{\infty}(\Omega)}
$$

and $\lim _{k \rightarrow \infty} \varphi_{k}(\boldsymbol{z})=\varphi(\boldsymbol{z})$ for $\boldsymbol{z}$ in $\Omega$. Thus $A(\Omega)$ is dense in $H^{\infty}(\Omega)$ in the bpwltopology.

The arguments given in Section 3 carry over to the algebra of multipliers and hence to $H^{\infty}(\Omega)$ in case $A(\Omega)$ is strongly dense in the bpwl-topology. We will state the results in one case and comment only on any necessary changes.

Definition 5.4. A domain $\Omega$ for which $A(\Omega)$ is bpwl-dense in $H^{\infty}(\Omega)$ is weakly pointed at $\alpha$ in the Šilov boundary of $M_{H^{\infty}(\Omega)}$ if $H^{\infty}(\Omega)$ is pointed at $\alpha$.

Again Izzo's result applies to show that $H^{\infty}(\Omega)$ is pointed at all p-points, which forms a dense subset of the Šilov boundary.

Let $\Omega$ be a bounded domain in $\mathbb{C}^{n}$ for which $A(\Omega)$ is strongly bpwl-dense in $H^{\infty}(\Omega)$. If $\mathscr{R}$ is a finite-rank quasi-free Hilbert module over $A(\Omega)$, then $\mathscr{R}$ is a contractive Hilbert module over $H^{\infty}(\Omega)$. Let $\mathfrak{T}_{\infty}(\mathscr{R})$ be the $\mathrm{C}^{*}$-algebra generated by $\left\{M_{\varphi}: \varphi \in H^{\infty}(\Omega)\right\}$, and let $\mathfrak{C}_{\infty}(\mathscr{R})$ be the commutator ideal in $\mathfrak{T}_{\infty}(\mathscr{R})$. Then 
$\mathfrak{T}_{\infty}(\mathscr{R}) / \mathfrak{C}_{\infty}(\mathscr{R}) \simeq C\left(X_{\mathscr{R}}^{\infty}\right)$ for a subset $X_{\mathscr{R}}^{\infty}$ of $M_{H^{\infty}(\Omega)}$. Finally, let $\Gamma_{\infty}$ be the restriction of the GBT to $\mathfrak{T}_{\infty}(\mathscr{R})$ and $\sigma_{\infty}$ the $*$-homomorphism from $\mathfrak{T}_{\infty}(\mathscr{R})$ to $C\left(X_{\mathscr{R}}^{\infty}\right)$.

Theorem 5.5. Let $\Omega$ be a bounded domain in $\mathbb{C}^{n}$ for which $A(\Omega)$ is strongly bpwldense in $H^{\infty}(\Omega)$. If $\Re$ is a finite-rank quasi-free Hilbert module over $A(\Omega)$, then the GBT defines a $*$-homomorphism from $\mathfrak{T}_{\infty}(\mathscr{R})$ onto $\mathfrak{C}\left(\partial H^{\infty}(\Omega)\right)$ whose kernel contains $\mathfrak{C}_{\infty}(\Re)$.

Proof. The proof is the same as for Theorem 3.3 with $H^{\infty}(\Omega)$ replacing $A(\Omega)$. The same problem exists in this case regarding the possible continuity of $\Gamma_{\infty}$ on $\Omega \cup \partial H^{\infty}(\Omega)$.

The classic examples of the foregoing structure occur for $\Omega$ the unit disk and $\mathscr{R}$ the Hardy and Bergman modules. In [Douglas 1972], the second author established that the quotient $\mathfrak{T}_{\infty}\left(H^{2}(\mathbb{D})\right) / \mathfrak{C}_{\infty}\left(H^{2}(\mathbb{D})\right)$ is isometrically isomorphic to $L^{\infty}(\mathbb{T}) \simeq C\left(\partial H^{\infty}(\mathbb{D})\right)$ via the symbol map which coincides on $\partial H^{\infty}(\mathbb{D})$ with the GBT. Hence, in this case the extension of the GBT restricted to $\partial H^{\infty}(\mathbb{D})$ coincides with the symbol map and the Berezin nullity coincides with $\mathfrak{C}_{\infty}\left(H^{2}(\mathbb{D})\right.$ ) (see [Axler and Zheng 2004]). The result of McDonald and Sundberg [1979] shows that $\mathfrak{T}_{\infty}\left(B^{2}(\mathbb{D})\right) / \mathfrak{C}_{\infty}\left(B^{2}(\mathbb{D})\right)$ is isometrically isomorphic to $C\left(M_{1}\right)$, where $M_{1}$ is the subset of $M_{H^{\infty}(\mathbb{D})}$ consisting of the one-point parts. Since $\partial H^{\infty}(\mathbb{D}) \subsetneq M_{1}$, we see in this case that the kernel of the extension of the GBT restricted to $\partial H^{\infty}(\mathbb{D})$ does not equal $\left.\mathfrak{C}_{\infty}\left(B^{2}(\mathbb{D})\right)\right)$.

There is a natural family of kernel Hilbert spaces $\mathscr{R}_{n}$ on $\mathbb{D}$ which define quasifree Hilbert modules over $A(\mathbb{D})$. The kernel functions are $(1-z \bar{\omega})^{-(n+1)}$ and the first two are the Hardy and Bergman modules. A natural problem is to determine the maximal ideal space of $\mathfrak{T}_{\infty}\left(\mathscr{R}_{n}\right) / \mathfrak{C}_{\infty}\left(\mathscr{R}_{n}\right)$ which can be identified with a closed

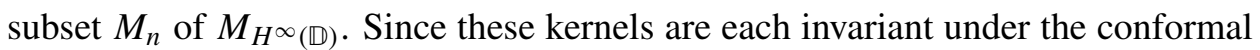
self-maps of $\mathbb{D}$, it follows that the maximal ideal spaces $M_{n}$ are also. (ADDED IN PROOF: D. Zheng reports that he has shown that $M_{n}=M_{1}$ for all $n \geq 1$.)

Another interesting question would seem to be the characterization of all conformally invariant closed subsets of $M_{H^{\infty}(\mathbb{D})}$, or at least the ones that are the maximal ideal space of subalgebras between $H^{\infty}(\mathbb{D})$ and $L^{\infty}(\mathbb{T})$ or, equivalently, the conformally invariant Douglas algebras. Known examples of the latter are $H^{\infty}(\mathbb{D})$, $H^{\infty}(\mathbb{D})+C, A_{\text {int }}$ (the algebra generated by $H^{\infty}(\mathbb{D})$ and the complex conjugate of the interpolating Blaschke products) and $L^{\infty}(\mathbb{T})$, with the corresponding maximal ideal spaces being $M_{H^{\infty}(\mathbb{D})}, M_{H^{\infty}(\mathbb{D})} \backslash \mathbb{D}, M_{1}, \partial H^{\infty}(\mathbb{D})$. An example not on this list is obtained from the Douglas algebra generated by $H^{\infty}(\mathbb{D})$ and the complex conjugates of the singular inner functions for purely atomic measures. It would be of interest to better understand this algebra. Actually, it may be better to consider 
those Douglas algebras which are invariant under the full automorphism group of $H^{\infty}(\mathbb{D})$.

\section{Multiplication operators bounded below}

In this final section we want to relate further the commutator ideal and the Berezin nullity to the nature of the multiplication operators. We have already considered one aspect of such a relationship in Theorem 4.1 and the paragraphs that follow. There we considered the implication of the operators $M_{z-z_{0}}$ having closed range for the case of $\Omega$ a planar domain. We want to consider this matter in more detail and restrict attention, at first, to the case of the unit disk. We first recall that the question can be reduced to that of inner functions.

Lemma 6.1. Let $\Re$ be a finite-rank quasi-free Hilbert module over $A(\mathbb{D})$, let $\varphi \in$ $H^{\infty}(\mathbb{D})$ such that $\varphi=\theta f$, where $\theta$ is inner and $f$ is outer. The operator $M_{\varphi}$ has closed range if and only if $f$ is invertible in $H^{\infty}(\mathbb{D})$ and $M_{\theta}$ has closed range.

Proof. First observe that if $f$ is an outer function, then $M_{f}$ has dense range. In [Garnett 1981, Theorem II.7.4] it is shown that if $f$ is outer, there are functions $g_{n} \in H^{\infty}(\mathbb{D})$ so that $\left\|f g_{n}\right\|_{\infty} \leq 1$ and $f g_{n}$ converges to 1 almost everywhere on the unit circle. Since point evaluation in the disk is absolutely continuous, and the kernels are continuous on $\mathbb{D}$, it follows that $f(z) g_{n}(z)$ converges pointwise to 1 ; and in fact it converges uniformly on compact subsets of $\mathbb{D}$. In particular it converges in the bpwl topology, and so it follows from Lemma 5.1 that $M_{f g_{n}}$ converges to the identity in the weak operator topology. As the range of $M_{f}$ contains the range of $M_{f g_{n}}$ for all $n \geq 1$, one deduces that $M_{f}$ has dense range.

Since $M_{\varphi}=M_{\theta} M_{f}$ has closed range, the operator is bounded below because it is one-to-one. Thus $M_{f}$ is bounded below. Since it has dense range, $M_{f}$ is invertible; and so $M_{\theta}$ has closed range.

Consequently $M_{f}^{*}$ is invertible, and hence bounded below. So

$$
\left\|\overline{f(z)} k_{z}\right\|=\left\|M_{f}^{*} k_{z}\right\| \geq \varepsilon\left\|k_{z}\right\|
$$

for every vector $k_{z}$ in the range of $V_{z}$, whence $|f(z)| \geq \varepsilon$ for $z \in \mathbb{D}$, and so $1 / f$ is in $H^{\infty}(\mathbb{D})$.

Proposition 6.2. Let $\mathscr{R}$ be a finite-rank quasi-free Hilbert module over $A(\mathbb{D})$ and let $\varphi$ be in $H^{\infty}(\mathbb{D})$. If $M_{\varphi}$ has closed range, then $\widehat{\varphi}$ is nonzero on $X_{\mathscr{R}}^{\infty}$.

Proof. Since $M_{\varphi}$ is one-to-one, $M_{\varphi}$ has closed range if and only if $M_{\varphi}^{*} M_{\varphi}$ is an invertible operator. In that case, it is invertible in $\mathfrak{T}_{\infty}(\mathscr{R})$ and hence its image, $\left.\widehat{\varphi}\right|_{X_{\Re}^{\infty}}$, is invertible in $C\left(X_{\mathscr{R}}^{\infty}\right)$ which completes the proof.

An interesting question is whether the converse of this result holds, that is, whether the maximal ideal space of $\mathfrak{T}_{\infty}(\mathscr{R}) / \mathfrak{C}_{\infty}(\mathscr{R})$ is determined by where the 
Gelfand transforms of the functions in $H^{\infty}(\mathbb{D})$ whose multipliers have closed range, are nonzero. First, the converse holds for $\mathscr{R}=H^{2}(\mathbb{D})$. The argument proceeds as follows: Multiplication by each inner function defines an isometry on $H^{2}(\mathbb{D})$ and hence has closed range. Finally, the Šilov boundary of $H^{\infty}(\mathbb{D})$ consists precisely of those points in $M_{H^{\infty}(\mathbb{D})}$ for which the Gelfand transforms of all inner functions don't vanish. In the case $\mathscr{R}=B^{2}(\mathbb{D})$, Horowitz [1977] and McDonald and Sundberg [1979] have shown that the same thing holds, that is, $X_{B^{2}(\mathbb{D})}^{\infty}$ is precisely the subset of $M_{H^{\infty}}(\mathbb{D})$ on which an inner function is nonzero precisely when as a module multiplier it has closed range. Moreover, they showed that the inner functions with closed range on $B^{2}(\mathbb{D})$ are precisely the finite products of interpolating Blaschke products.

Also, one might hope that closedness of the range for $M_{\varphi}$ could be inferred directly from the Gelfand transform (or the Berezin transform) of the function $\varphi$. The preceding analysis shows that is the case for $\mathscr{R}=B^{2}(\mathbb{D})$ if one considers the asymptotic behavior of the transform on $M_{H^{\infty}(\mathbb{D})}$ in the directions of $X_{B^{2}(\mathbb{D})}^{\infty}$ or at limit nets that converge to the one-point parts of $M_{H^{\infty}(\mathbb{D})}$. There is another more direct possibility, however. If $M_{\varphi}$ has closed range, then $\left\|M_{\varphi} k_{z}\right\| \geq \varepsilon\left\|k_{z}\right\|$ for some $\varepsilon>0$ and all $z$ in $\mathbb{D}$, where $k_{z}$ is in the range of $V_{z}$. Is the converse true? That is, is it enough to test whether $M_{\varphi}$ is bounded below on just these vectors? This is trivially the case for $\mathscr{R}=H^{2}(\mathbb{D})$. Zhu has informed us that this is the case for $\mathscr{R}=B^{2}(\mathbb{D})$ for $\varphi$ an inner function. His proof rests on the characterization of the inner functions on $B^{2}(\mathbb{D})$ with closed range. What about for other quasi-free Hilbert modules over $A(\mathbb{D})$ ?

If one examines the proof of the preceding proposition, one sees that it extends to a more general setting with no change.

Proposition 6.3. Let $\Omega$ be a bounded domain in $\mathbb{C}^{n}$. For $\mathscr{R}$ a finite-rank quasi-free Hilbert module over $A(\Omega)$ and $\varphi$ in $H^{\infty}(\Omega)$, a necessary condition for $M_{\varphi}$ to have closed range is for $\left.\widehat{\varphi}\right|_{X_{\Re}^{\infty}}$ to be invertible in $C\left(X_{\Re}^{\infty}\right)$.

Sundberg [1987] obtains a converse to this result under somewhat different but related assumptions. In particular, he assumes that $\mathscr{R}$ is the compression of a larger module $N$ on which the module action of $A(\Omega)$ is via normal operators. We adapt his proof to our context but at the price of having to consider finite columns of operators defined on $\mathscr{R}$ by module multiplication or their adjoints.

Based on Theorem 5.5 we can assume $\sigma_{\infty}$ is defined on the whole Šilov boundary of $M_{H^{\infty}(\Omega)}$. The following result is an extension of Theorem 4.3 to the case when there are not finitely many generators.

Theorem 6.4. Let $\Omega$ be a bounded domain in $\mathbb{C}^{n}$, and let $\mathscr{R}$ be a finite-rank quasifree Hilbert module over $A(\Omega)$. A point $\alpha$ in $M_{H^{\infty}(\Omega)}$ is in $X_{\mathscr{R}}^{\infty}$ if and only if all 
operators of the form

$$
T=\left[\begin{array}{c}
T_{1} \\
\vdots \\
T_{n}
\end{array}\right]
$$

mapping $\mathscr{R}$ to $\mathscr{R}^{(n)}$ with $T_{i}=M_{\varphi_{i}}$ or $M_{\varphi_{i}}^{*}$ for $\varphi_{i}$ in $H^{\infty}(\Omega)$ satisfying $\widehat{\varphi}_{i}(\alpha)=0$ have nonclosed range.

Proof. Fix $\alpha$ in $X_{\mathscr{R}}^{\infty}$, and let $\Phi$ be the character on $\mathfrak{T}_{\infty}(\mathscr{R})$ extending evaluation at $\alpha$. Consider an operator

$$
T=\left[\begin{array}{c}
T_{1} \\
\vdots \\
T_{k}
\end{array}\right]
$$

mapping $\mathscr{R}$ to $\mathscr{R}^{(k)}, k$ copies of $\mathscr{R}$, where each $T_{i}=M_{\varphi_{i}}$ or $M_{\varphi_{i}}^{*}$ for $\varphi_{i}$ in $H^{\infty}(\Omega)$ satisfying $\widehat{\varphi}_{i}(\alpha)=0$. Suppose that $T$ has closed range. Let $\psi$ be a function in $H^{\infty}(\Omega)$ for which $\widehat{\psi}(\alpha)=0$, and consider the operator $T^{\prime}=\left[\begin{array}{c}T \\ M_{\psi}\end{array}\right]$ from $\mathscr{R}$ to $\mathscr{R}^{(k+1)}$. Then $T^{\prime}$ has closed range and is one-to-one since $M_{\psi}$ is one-to-one. Therefore, the operator $T^{\prime *} T^{\prime}=\sum_{i} T_{i}^{*} T_{i}+M_{\psi}^{*} M_{\psi}$ is invertible. Hence we obtain a contradiction:

$$
\begin{aligned}
0 \neq \Phi\left(T^{* *} T^{\prime}\right)(\alpha) & =\sum_{i} \Phi\left(T_{i}^{*} T_{i}\right)(\alpha)+\Phi\left(M_{\psi}^{*} M_{\psi}\right)(\alpha) \\
& =\sum_{i}\left|\widehat{\varphi}_{i}(\alpha)\right|^{2}+|\widehat{\psi}(\alpha)|^{2}=0
\end{aligned}
$$

Now suppose $\alpha$ is a point in $M_{H^{\infty}(\Omega)}$ satisfying the statement of the theorem, that is, all operators of the form

$$
T=\left[\begin{array}{c}
T_{1} \\
\vdots \\
T_{n}
\end{array}\right]
$$

mapping $\mathscr{R}$ to $\mathscr{R}^{(n)}$ with $T_{i}=M_{\varphi_{i}}$ or $M_{\varphi_{i}}^{*}$ for $\varphi_{i}$ in $H^{\infty}(\Omega)$ satisfying $\widehat{\varphi}_{i}(\alpha)=0$ have nonclosed range. Hence we may construct a net of unit vectors $\left\{x_{\lambda}\right\}$ indexed by finite subsets of $H^{\infty}(\Omega)$ as follows. Let $T_{\lambda}$ be the column operator with $2|\lambda|$ entries $T_{\varphi-\widehat{\varphi}(\alpha)}$ and $T_{\varphi-\widehat{\varphi}(\alpha)}^{*}$ for $\varphi \in \lambda$. Since this is not bounded below, select a unit vector $x_{\lambda}$ so that $\left\|T_{\lambda} x_{\lambda}\right\|<|\lambda|^{-1}$. By Lemma 3.9, there are no reducing eigenvalues. So it is easy to verify that the net $x_{\lambda}$ tends weakly to 0 .

Now following the proof of Theorem 4.3, we can show that the projections $P_{\lambda}$ onto the span of $x_{\lambda}$ asymptotically reduce each $M_{\varphi}$, and hence all of $\mathfrak{T}_{\infty}(\mathscr{R})$. It therefore follows as in that proof that $\Phi(A)=\lim _{\lambda}\left(A x_{\lambda}, x_{\lambda}\right)$ is a character extending evaluation at $\alpha$. 
Sundberg [1987] using the additional structure present in his context, is able to replace the column operator by a single operator. In his case, there is a Hilbert super-module $\mathcal{N}$ over $A(\Omega)$ containing the given module $\mathscr{R}$ for which all operators $N_{\varphi}$ defined by module multipliers $\varphi$ in $A(\Omega)$ (or, equivalently, in $H^{\infty}(\Omega)$ if $\Omega$ is weak pointed) are normal. If $\mathfrak{A}_{\mathcal{N}}$, respectively $\mathfrak{A}_{\mathcal{N}}^{\infty}$, is the $\mathrm{C}^{*}$-algebra generated by $\left\{N_{\varphi}: \varphi \in A(\Omega)\right\}$, or $\left\{N_{\varphi}: \varphi \in H^{\infty}(\Omega)\right\}$, then $\mathfrak{A}_{\mathcal{N}} \simeq C\left(Y_{\mathcal{N}}\right)$ and $\mathfrak{A}_{\mathcal{N}}^{\infty} \simeq C\left(Y_{\mathcal{N}}^{\infty}\right)$. Consideration of the maps from $A(\Omega)$ onto $\mathfrak{A}_{\mathcal{N}}$ and $H^{\infty}(\Omega)$ into $\mathfrak{A}_{\mathcal{N}}^{\infty}$ allows one to identify

$$
\partial A(\Omega) \subseteq Y_{\mathcal{N}} \subseteq M_{A(\Omega)} \quad \text { and } \quad \partial H^{\infty}(\Omega) \subseteq Y_{\mathcal{N}}^{\infty} \subseteq M_{H^{\infty}(\Omega)}
$$

Let $P$ be the projection of $\mathcal{N}$ onto $\mathscr{R}$. Consideration of the matrix representation for module multipliers in $A(\Omega)$ and $H^{\infty}(\Omega)$ relative to the decomposition $\mathcal{N}=$ $\mathscr{R} \oplus \mathscr{R}^{\perp}$ enables one to conclude that $\mathfrak{T}(\mathscr{R})$ and $\mathfrak{T}^{\infty}(\mathscr{R})$ coincide with the $\mathrm{C}^{*}$ algebras generated by the collections $\left\{T_{\varphi}: \varphi \in C\left(Y_{\mathcal{N}}\right)\right\}$ and $\left\{T_{\varphi}: \varphi \in L^{\infty}(\mu)\right\}$, where $T_{\varphi}$ is defined to be the "Toeplitz operator" $P N_{\varphi} P$ and $\mu$ is a scalar spectral measure for $\mathcal{N}$. Two obvious questions which now present themselves concern the relationship, if any, between $X_{\mathscr{R}}$ and $Y_{\mathscr{R}}$ and between $X_{\mathscr{R}}^{\infty}$ and $Y_{\mathscr{R}}^{\infty}$.

Example 6.5. Let $\mu$ be the positive measure on the closure of $\mathbb{D}$ defined as the sum of Lebesgue measure $d \theta$ on $\partial \mathbb{D}$ plus the atomic measure of mass one supported at the origin of $\mathbb{D}$. If $H^{2}(\mu)$ is the closure of $A(\mathbb{D})$ in $L^{2}(\mu)$, then it is a rank-one quasi-free Hilbert module over $A(\mathbb{D})$. One can see that $Y_{H^{2}(\mu)}$ is $\{0\} \cup \partial \mathbb{D}$, while

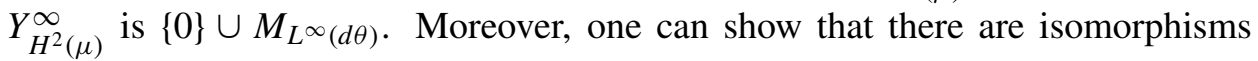
$\mathfrak{T}\left(H^{2}(\mathbb{D})\right) \cong \mathfrak{T}\left(H^{2}(\mu)\right)$ and $\mathfrak{T}^{\infty}\left(H^{2}(\mathbb{D})\right) \cong \mathfrak{T}^{\infty}\left(H^{2}(\mu)\right)$, both preserving the respective inclusion maps of $A(\mathbb{D})$ and $H^{\infty}(\mathbb{D})$ into the $\mathrm{C}^{*}$-algebras. Therefore, we have $X_{H^{2}(\mu)}=\partial \mathbb{D}$ and $X_{H^{2}(\mu)}^{\infty}=\partial H^{\infty}(\Omega)$. Since both $X_{H^{2}(\mu)} \neq Y_{H^{2}(\mu)}$ and $X_{H^{2}(\mu)}^{\infty} \neq Y_{H^{2}(\mu)}^{\infty}$, we see that neither of the pairs $X_{\mathscr{R}}$ and $Y_{\mathscr{R}}$ nor $X_{\mathscr{R}}^{\infty}$ and $Y_{\mathscr{R}}^{\infty}$ are equal, in general. However, we can ask whether inclusion holds, that is, does $X_{\mathscr{R}} \subseteq Y_{\mathscr{R}}$ or $X_{\mathscr{R}}^{\infty} \subseteq Y_{\mathscr{R}}^{\infty}$ ?

\section{References}

[Agrawal and Salinas 1988] O. P. Agrawal and N. Salinas, "Sharp kernels and canonical subspaces (revised)", Amer. J. Math. 110:1 (1988), 23-47. MR 89g:47026 Zbl 0644.47029

[Arveson 1969] W. B. Arveson, "Subalgebras of $C^{*}$-algebras", Acta Math. 123 (1969), 141-224. MR 40:6274 Zbl 0194.15701

[Axler and Zheng 2004] S. Axler and D. Zheng, "Toeplitz algebras on the disk", preprint, 2004, Available at http://www.axler.net/ToeplitzAlgebras.html.

[Berezin 1972] F. A. Berezin, "Covariant and contravariant symbols of operators", Izv. Akad. Nauk SSSR Ser. Mat. 36 (1972), 1134-1167. MR 50:2996 Zbl 0259.47004

[Bunce 1971] J. Bunce, “The joint spectrum of commuting nonnormal operators", Proc. Amer. Math. Soc. 29 (1971), 499-505. MR 44:832 Zbl 0215.20903 
[Curto and Salinas 1984] R. E. Curto and N. Salinas, "Generalized Bergman kernels and the CowenDouglas theory”, Amer. J. Math. 106:2 (1984), 447-488. MR 85e:47042 Zbl 0583.47037

[Davidson 1996] K. R. Davidson, $C^{*}$-algebras by example, Fields Institute Monographs 6, American Mathematical Society, Providence, RI, 1996. MR 97i:46095 Zbl 0958.46029

[Douglas 1972] R. G. Douglas, Banach algebra techniques in operator theory, Pure and Applied Math. 49, Academic Press, New York, 1972. MR 50:14335 Zbl 0247.47001

[Douglas and Misra 2003] R. G. Douglas and G. Misra, "Quasi-free resolutions of Hilbert modules", Integral Equations Operator Theory 47:4 (2003), 435-456. MR 2004i:46109 Zbl 02078293

[Gamelin 1969] T. W. Gamelin, Uniform algebras, Prentice-Hall, Englewood Cliffs, NJ, 1969. MR 53:14137 Zbl 0213.40401

[Garnett 1981] J. B. Garnett, Bounded analytic functions, Pure and Applied Mathematics 96, Academic Press, New York, 1981. MR 83g:30037 Zbl 0469.30024

[Hedenmalm et al. 2000] H. Hedenmalm, , B. Korenblum, and K. Zhu, Theory of Bergman spaces, Graduate Texts in Mathematics 199, Springer, New York, 2000. MR 2001c:46043 Zbl 0955.32003

[Horowitz 1977] C. Horowitz, "Factorization theorems for functions in the Bergman spaces", Duke Math. J. 44:1 (1977), 201-213. MR 55:681 Zbl 0362.30031

[Izzo 2005] A. Izzo, “The linear span of peak functions", Proc. Edinburgh Math. Soc. (2005). To appear.

[McDonald et al. 1979] G. McDonald, , and C. a. Sundberg, "Toeplitz operators on the disc", Indiana Univ. Math. J. 28:4 (1979), 595-611. MR 80h:47034 Zbl 0439.47022

[Salinas 1988] N. Salinas, "Products of kernel functions and module tensor products", pp. 219241 in Topics in operator theory: Constantin Apostol memorial issue, edited by I. Gohberg, Oper. Theory Adv. Appl. 32, Birkhäuser, Basel, 1988. MR 90a:46061 Zbl 0669.46016

[Shields 1974] A. L. Shields, "Weighted shift operators and analytic function theory", pp. 49-128 in Topics in operator theory, edited by C. Pearcy, Math. Surveys 13, Amer. Math. Soc., Providence, R.I., 1974. MR 50:14341 Zbl 0303.47021

[Sundberg 1987] C. Sundberg, "Exact sequences for generalized Toeplitz operators", Proc. Amer. Math. Soc. 101:4 (1987), 634-636. MR 89e:47040 Zbl 0642.47012

[Voiculescu 1976] D. Voiculescu, “A non-commutative Weyl-von Neumann theorem”, Rev. Roumaine Math. Pures Appl. 21:1 (1976), 97-113. MR 54:3427 Zbl 0335.46039

Received February 13, 2004. Revised March 24, 2005.

\author{
KENNETH R. DAVIDSON \\ Pure Mathematics Department \\ UNIVERSITY OF WATERLOO \\ WATERLOO \\ ONTARIO N2L-3G1 \\ CANADA
}

krdavids@uwaterloo.ca

Ronald G. DOUgLaS

MATHEMATICS DEPARTMENT

TEXAS A\&M UNIVERSITY

College Station

TEXAS 77843-3368

UNITED STATES

rgd@tamu.edu 